\title{
Analysis of Acoustic Space Variability in Speech Affected by
}

\section{Depression}

\author{
Nicholas Cummins ${ }^{\mathrm{a}, \mathrm{b},{ }^{*},}$, Vidhyasaharan Sethu ${ }^{\mathrm{a}}$, Julien Epps ${ }^{\mathrm{a}, \mathrm{b},{ }^{*},}$ \\ Sebastian Schnieder ${ }^{\mathrm{d}, \mathrm{e}}$, Jarek Krajewski ${ }^{\mathrm{d}, \mathrm{e}}$ \\ ${ }^{a}$ School of Elec. Eng. and Telecomm., The University of New South Wales, Sydney Australia \\ ${ }^{\mathrm{b}}$ ATP Research Laboratory, National ICT Australia (NICTA), Australia \\ ${ }^{d}$ Experimental Industrial Psychology, University of Wuppertal, Wuppertal, Germany \\ ${ }^{\mathrm{e}}$ Industrial Psychology, Rhenish University of Applied Sciences Cologne, Germany
}

\begin{abstract}
The spectral and energy properties of speech have consistently been observed to change with a speaker's level of clinical depression. This has resulted in spectral and energy based features being a key component in many speech-based classification and prediction systems. However there has been no in-depth investigation into understanding how acoustic models of spectral features are affected by depression. This paper investigates the hypothesis that the effects of depression in speech manifest as a reduction in the spread of phonetic events in acoustic space as modelled by Gaussian Mixture Models (GMM) in combination with Mel Frequency Cepstral Coefficients (MFCC). Our investigation uses three measures of acoustic variability: Average Weighted Variance (AWV), Acoustic Movement (AM) and Acoustic Volume, which attempt to model depression specific acoustic variations (AWV and Acoustic Volume), or the trajectory of a speech in the acoustic space (AM). Within our analysis we present the Probabilistic Acoustic Volume (PAV) a novel method for robustly estimating Acoustic Volume using a Monte Carlo sampling of the feature distribution being
\end{abstract}

\footnotetext{
* Corresponding Author

Tel.: + 61293856579 (Epps)

E-mail addresses: n.p.cummins@unsw.edu.au (Cummins), j.epps@unsw.edu.au (Epps)
} 
modelled. We show that using an array of PAV points we gain insights into how the concentration of the feature vectors in the feature space changes with depression. Key results - found on two commonly used depression corpora - consistently indicate that as a speaker's level of depression increases there are statistically significantly reductions in both AWV $\left(-0.44 \leq r_{s} \leq-0.18\right.$ with $\left.p<.05\right)$ and AM $\left(-0.26 \leq r_{s} \leq-0.19\right.$ with $\left.p<.05\right)$ values, indicating a decrease in localised acoustic variance and smoothing in acoustic trajectory respectively. Further there are also statistically significant reductions $\left(-0.32 \leq r_{s} \leq-0.20\right.$ with $\left.p<.05\right)$ in Acoustic Volume measures and strong statistical evidence $\left(-0.48 \leq r_{s} \leq-0.23\right.$ with $\left.p<.05\right)$ that the MFCC feature space becomes more concentrated. Quantifying these effects is expected to be a key step towards building an objective classification or prediction system which is robust to many of the unwanted - in terms of depression analysis sources of variability modulated into a speech signal.

\section{Keywords}

Depression, Objective Diagnosis, Gaussian Mixture Models, Maximum a Posteriori Adaption, Mel Frequency Cepstral Coefficient's, Acoustic Variability, Acoustic Volume 


\section{Introduction}

\subsection{Societal Cost of Depression}

Clinical depression has been identified by the World Health Organization (WHO) as a leading cause of disability worldwide (Kessler et al., 2003; Ustun, 2004). Depression leads to high direct (health system related) costs of medical treatment, preventive treatment, rehabilitation measure, and care measures. Olesen et al. (2012) estimated that there were at least 30.3 million people affected by depression across European Union in 2010. Further they estimated direct health care costs per patient of $€ 797$, indirect health care medical costs (e.g. transportation to treatment) of $€ 464$ and indirect costs (e.g. lost productivity) of $€ 1782$. The total cost of depression in the European Union in 2010 was estimated to be $€ 92$ billion (Olesen et al., 2012). In Germany alone it has been estimated that the direct medical costs of depression are $€ 5.2$ billion per year (Allianz Deutschland and RWI, 2011). Further indirect costs due to mortality (€1.3 Billion), permanent invalidity ( $€ 4.6$ Billion), sick leave (€1.6 Billion) and lost productivity (€9.3 Billion) were also observed leading to estimated total costs of $€ 22$ Billion (Allianz Deutschland and RWI, 2011).

Research into depression diagnosis and monitoring methods can potentially help reduce these costs. Improved diagnostic paradigms could lead to therapeutic devices which provide immediate feedback or automatically contact triage services during acute depressive phases. Ambulatory (Outpatient) Depression Monitoring could provide a cheap and effortless screening tool for early detection of depressive states leading to improved primary, secondary and tertiary prevention strategies (World Health Organisation, 2004). New fields of depression research could be opened up, such as tools which enable longitudinal research into the impacts and causality of real-life events on depression and /or the fine grained evaluation of therapy. 


\subsection{State-of-the-art Diagnostic Methods}

Currently state-of-the-art diagnostic methods are time consuming and subjective. The current edition of the widely used Diagnostic and Statistical Manual of Mental Disorders (DSM-V) defines clinical depression as either depressed mood or diminished interest in combination with four or more of the symptoms listed in Table 1. Using this definition, Østergaard et al. (2011) calculated that there are at least 1497 possible unique profiles of depression; this variation introduces a high degree of complexity when trying to fit the clinical profile of a depressed individual into an objective level (Mitchell et al., 2009). A range of screening tools based on patient responses to clinician-led or selfcompleted questionnaires exist to help elicit and score these behaviours. However, since many of these key symptoms of depression, such as low mood, are not physical in nature and therefore not directly measurable, these tests are open to a range of subjective biases and in the clinician-led cases require a large degree of clinical training to produce acceptable results (Blais and Baer, 2010; Mundt et al., 2007).

Table 1: Symptoms associated with Depression (American-Psychiatric-Association, 2013)

Depressed Mood and/or Markedly diminished interest or pleasure

\section{In combination with four of}

Psychomotor retardation OR agitation

Diminished ability to think/concentrate OR Increased indecisiveness

Fatigue OR Loss of energy

Insomnia OR hypersomnia

Significant weight loss OR weight gain

Feelings of worthlessness OR Excessive / inappropriate guilt

Recurrent thoughts of death OR Recurrent suicidal ideation

\subsection{Biomarkers and Behavioural Signals for Depression}

Whilst current diagnostic measures remain rooted in these subjective and often time consuming assessment methodologies, research into finding a set of measurable biomarkers or observable 
behavioural signals to improve diagnosis accuracy and treatment efficacy is gaining in popularity. Research into biological markers for depression has revealed several promising results including genetic abnormalities (Schmidt et al., 2011), biochemical markers such plasma proteins (Domenici et al., 2010) and both wake and sleep electroencephalogram (Steiger and Kimura, 2010). However, to date, no true biomarker for depression has been found (Schmidt et al., 2011). Many represent a marker for an individual at risk of suffering a depressive episode.

Very recent research shows promise in use of social signal processing of behavioural signals, as a diagnostic and monitoring aid for depression (Cummins et al., 2013c; Girard et al., 2013; Joshi et al., 2013; Scherer et al., 2014; Williamson et al., 2014). Speech is considered a key component in any behavioural based diagnostic tool (Cummins et al., 2015). Speech affected by depression is often characterised by decreases in verbal activity; decreases in utterance length, and a reduction in speech rate and long silent pauses (Balsters et al., 2012; Hall et al., 1995; Sobin and Sackeim, 1997). The heterogeneous nature of depression induces a range of different physiological effects which alter speech motor control. Cognitive impairments slow speech planning and preparation of neuromuscular commands needed to produce speech, whilst changes in affective state, fatigue and psychomotor retardation affect muscle tension, creating articulatory errors and altering vocal tract properties (Christopher and MacDonald, 2005; Flint et al., 1993).

Many of the commonly observed symptoms of depression, such as a reduced cognitive ability, a continuous negative affect, fatigue and psychomotor retardation, cause neuromotor disturbances (Caligiuri and Ellwanger, 2000). These disturbances, often observable as a reduction in facial and head movement variability (Girard et al., 2013; Scherer et al., 2014), affect muscle control and tension (Caligiuri and Ellwanger, 2000; Sobin and Sackeim, 1997). In speech, these changes have been shown to manifest as a reduction in either energy (intensity) variance (Kuny and Stassen, 1993; Quatieri and Malyska, 2012) or, as our recent results have indicated, a consistent trend of negative correlations in sub-band energy variability with increasing levels of depression (Cummins et al., 
2013a, 2013b). Intuitively this decrease in variability matches the descriptions of depressed speech as expressionless.

However the variance of frame-level spectral features is not ideal for classifying or predicting a speaker's level of depression as spectral features are representative of all the information contained in speech, linguistic and paralinguistic. As a result of this the parametric modelling of spectral-based features is a very popular approach when classifying or predicting a speaker's level of depression (Cummins et al., 2013b, 2013c, 2011; Sturim et al., 2011; Williamson et al., 2013). However, despite their wide use there has been no in-depth investigation into understanding how the acoustic models, on which these classification and prediction systems are based, are affected by this reported lack of spectral variability and other alterations in speech motor control.

\subsection{Depression and Acoustic Space}

The focus of this paper is on identifying depression specific changes in acoustic models. Specifically, changes to the generalised distribution of phonetic events in a suitable feature space, referred to herein as the acoustic space. Given speech affected by depression has been consistently linked with decreases spectral and energy based feature variability, we hypothesise that the effects of depression manifest as a reduction in the spread of phonetic events in acoustic space. Our preliminary investigations into this hypothesis indicate that increases in a speaker's level of depression can be characterised by a decrease in both local (Cummins et al., 2013b) and global (Cummins et al., 2014b) variability in the acoustic space represented by a Gaussian mixture models of the Mel Frequency Cepstral Coefficient feature space. However both sets of results (from the investigations of local variability and global variability) are published on separate corpora and are not straightforward to generalise - hence the experiments presented in this paper are conducted over two commonly used depression corpora.

The rest of this paper is laid out as follows; Section 2 expands on this aim and hypothesis; highlighting the need for investigations into how depression manifests in acoustic space and 
introducing the measures of acoustic variability used in this paper. Section 3 presents the two depression corpora and main settings used to find all experimental results reported in this paper. Section 4 presents both an in-depth explanation of all acoustic measures used in this paper as well as a set of experimental results and analysis for each feature. Section 5 presents a comparison of a subset of results between both corpora and speculates on the importance of choosing a suitable paradigm when collecting speech samples for depression detection. Finally a succinct conclusion of the work presented in this paper is offered in Section 6.

\section{Acoustic Space Analysis}

\subsection{Alterations in Speech Motor Control}

A wide range of prosodic, source, formant and spectral alterations have consistently been attributed to depression (Table 2). However, these features were never designed to capture depression-based information from speech. Therefore, given the complexity of speech production, it does seem reasonable to assume that the performance of any classification system based on just one of these attributes will be somewhat limited. An example of this limitation can be highlighted using the Normalised Amplitude Quotient (NAQ) feature presented in (Scherer et al., 2013). Whilst NAQ shows significant increase in depressed speakers when compared to non-depressed speakers, it was originally designed to differentiate between breathy and tense voice qualities (Alku et al., 2002). Therefore a speaker with a breathy voice, due to reasons unrelated to depression, could present as a false positive in a classifier based purely on NAQ. Similarly errors could be introduced to a prosodic based classifier due simply due to natural variations in speaking style (Nilsonne et al., 1987; Stassen et al., 1991).

However using this same set of effects (Table 2), we can infer that depression produces a wide range of alterations in speech motor control, i.e. the significant increase in NAQ seen with increasing levels of speaker depression provides strong evidence for alterations in vocal fold coordination with 
increasing levels of speaker depression. Speech motor control is the terminology for the muscular systems and strategies needed to control speech production (Kent, 2000). The input to this speech motor system is typically assumed to be an intended phonetic sequence, which is stored briefly in working memory before the speaker executes the neuromuscular commands to initiate the required speech motor actions. Working memory impairments are commonly associated with depression (Christopher and MacDonald, 2005; Joormann and Gotlib, 2008) and this could be characterised by increased speech pause duration, commonly reported for depressed speech (Alpert et al., 2001; Mundt et al., 2012, 2007; Stassen et al., 1998).

The output of the speech motor system is considered to be the articulatory movements needed to convey an intended phonetic sequence. The main articulators include the mandible, lip, tongue, velum and pharyngeal constrictors, all of which play a part in determining the shape of the vocal tract. Reductions in both energy and formant dynamics provide evidence for increases in articulatory effort with increasing levels of speaker depression (Helfer et al., 2013; Kuny and Stassen, 1993; Quatieri and Malyska, 2012; Williamson et al., 2013). A consistent trend of negative correlations in sub-band energy variability observed with increasing levels of depression provides evidence of changes to the resonance properties of the vocal tract (Cummins et al., 2013a, 2013b). 
Table 2: Evidence for a lack of speech motor control with increased level of speaker depression; $\downarrow$ indicates a reduction in the effect whilst $\uparrow$ indicates an increase the effect

\begin{tabular}{|c|c|c|c|}
\hline Feature & $\begin{array}{c}\text { Effect } \\
\text { Direction }\end{array}$ & Reference & $\begin{array}{l}\text { Inferred Speech } \\
\text { Motor Control } \\
\text { Alteration }\end{array}$ \\
\hline Pitch $^{\mathrm{a}}\left(F_{0}\right)$ variability & $\downarrow$ & $\begin{array}{l}\text { Nilsonne et al.(1987) } \\
\text { Stassen et al.(1995) }\end{array}$ & $\begin{array}{l}\text { Increased vocal } \\
\text { fold tension }\end{array}$ \\
\hline Speech rate & $\downarrow$ & Mundt et al. (2012) & Slowing of \\
\hline Voice onset time & $\uparrow$ & Flint et al. (1993) & articulatory muscles \\
\hline Jitter & $\uparrow$ & Quatieri and Malyska(2012) & Decreased vocal \\
\hline Shimmer & $\downarrow$ & Hönig et al.(2014) & fold coordination \\
\hline Normalised Amplitude Quotient & $\uparrow$ & Scherer et al. (2013) & \\
\hline Quasi Open Quotient & $\uparrow$ & Scherer et al. (2013) & \\
\hline Spirantization & $\uparrow$ & Flint et al. (1993) & Decreased \\
\hline Aspiration & $\uparrow$ & Quatieri and Malyska(2012) & articulator/laryngeal \\
\hline First Formant Location & $\uparrow$ & France et al. (2000) & coordination \\
\hline Second Formant Location & $\downarrow$ & Flint et al. (1993) & \\
\hline Relative shifts in spectral energy & $\uparrow$ & France et al. (2000) & Alterations in \\
\hline Energy Variability & $\uparrow$ & Quatieri and Malyska(2012) & vocal tract dynamics \\
\hline Sub-band Energy Variability & $\downarrow$ & Cummins et al. (2013a, 2013b) & \\
\hline
\end{tabular}

a) Rate of Vibration of the Vocal Folds

\subsection{Related Speech Motor Control Disorders}

Typical speech motor control disorders include; Dysarthria, Apraxia and Fluency Disorders such as stuttering (Kent, 2000). As they are speech disorders resulting from cognitive impairments associated with affect muscle control and tension (Kent, 2000), studies into dysarthric speech disorders are of particular interest for analysing speech affected by depression. Similarly to descriptions of speech affected by depression (Cummins et al., 2015), dysarthric speech disorders 
can be categorised by; intensity decay, prosodic abnormalities, speaking rate and abnormal formant patterns (Kent and Kim, 2003).

Dysarthria is common in individuals with Parkinson's disease (PD), speech affected by PD is often characterised by reduced articulatory movements and reduced vocal tract constriction (Sapir et al., 2010). Acoustic metrics used to measures speech alteration in individuals with PD include Vowel Space Area (VSA), Vowel Articulation Index (VAI) and Formant Centralization Ratio (FCR). VSA is usually constructed by the Euclidean distances between the F1 and F2 coordinates of the corner vowels /i/, /u/, and /a/ (Skodda et al., 2012). Significantly smaller vowel space is often reported in the literature when comparing speech affected by Parkinson's disease to speech from matched healthy subjects (McRae et al., 2002; Orozco-Arroyave et al., 2014; Sapir et al., 2010). However VSA is known to be sensitive to sensitive to inter-speaker variability, recording environment and method of formant extraction (Sapir et al., 2010). VAI and FCR are vowel production ratios and have been shown to be able to differentiate patients with PD and matched healthy controls (Sapir et al., 2010; Skodda et al., 2011). A major drawback when using either VSA, VAI or FCR is the time consuming nature of selecting suitable speech segments (Scherer et al., 2015); vowel tokens are often manually selected from recordings.

Flint et al. (1993) report acoustic similarities between depressed and PD patients including decreased second formant location and improper glottal closure. Flint et al. (1993) go on to speculate that these similarities are due to the prevalence of Psychomotor Retardation in both conditions. Initial studies into acoustic metrics applied to depressed speech show mixed results. Scherer et al. (2015) showed over a range of different corpora that speech affected by depression can be characterised by a reduced vowel space. However, Helfer et al. (2013) report that VAl and FCR performed poorly on low/high depression classification. Given acoustic similarities between speech affected by either depression or PD (Flint et al., 1993), as well as the reduced vowel space 
results presented in Scherer et al. (2015), we expected a reduction in our acoustic variability measures (Section 2.4) with increasing level of speaker depression.

\subsection{Acoustic Space Modelling}

Recent results show that information relating to depression alterations in speech motor control can be expected to be present in the speech spectrum (Cummins et al., 2013a). However the spectrum is a high dimensional representation of a speech signal with substantial redundancies. The use of Gaussian mixture models (GMM) in combination with Mel Frequency Cepstral Coefficients (MFCC) offers a robust way of representing the spectral content of an utterance, and has become the default method for (implicitly) partitioning (modelling) the acoustic space in many speech based systems. This paradigm is widely used in many different paralinguistic and vocal disorder classification tasks (Bocklet et al., 2013; Godino-Llorente et al., 2006; Schuller et al., 2013, 2011; Sethu et al., 2014). It has been shown to be suitable for classifying the presence or severity of depression (Alghowinem et al., 2012; Cummins et al., 2013b, 2011; Low et al., 2010; Sturim et al., 2011).

A GMM is a convex combination of Gaussian probability density functions:

$$
\mathcal{G}(\bar{x})=\sum_{i=1}^{M}{ }_{i} p\left(\bar{x} \mid \boldsymbol{\mu}_{\boldsymbol{i}}, \boldsymbol{\Sigma}_{\boldsymbol{i}}\right)
$$

where

$$
p\left(\bar{x} \mid \boldsymbol{\mu}_{\boldsymbol{i}}, \boldsymbol{\Sigma}_{\boldsymbol{i}}\right) \sim \mathcal{N}\left(\bar{x} ; \boldsymbol{\mu}_{\boldsymbol{i}}, \boldsymbol{\Sigma}_{\boldsymbol{i}}\right)
$$

in which $\mathcal{N}$ denotes the multivariate normal distribution, $\boldsymbol{\mu}_{\boldsymbol{i}}$ the mean vector, $\boldsymbol{\Sigma}_{\boldsymbol{i}}$ the covariance matrix and ${ }_{i}$ the prior probability or mixing weight for the $i$-th Gaussian distribution. Note: GMMs will be denoted by $\mathcal{G}$ in figures and equations. GMMs perform a soft clustering of the acoustic space - speech frames taken from similar phones (i.e. frames taken from two similar sounding phonemes such as /e/ and /æ//) should have similar mixture component posterior probabilities. The common 
assumption when using GMMs is that the means of the model relate to local average spectral shape and the covariance matrices to the extent of variations from the average shapes (Reynolds and Rose, 1995).

Trevino et al. (2011) report similarities in the size and manner of their correlations between both phoneme-length and phone-spread (energy based measure used to define variations in phoneme duration) with depression severity. Whilst their work does not focus specifically on a reduction of energy variability with increasing levels of depression - their analysis focused on changes in average phone duration - it does show that depression has similar effects on phonemes grouped together by manner of articulation. Combining both the reduction in energy and sub-band energy variation (Table 2), with the results presented by Trevino et al. (2011), it is not unreasonable to assume that both the spectral shape and variations, in particular, represented in the GMM will be affected by depression.

The most common method for using GMMs to model acoustic space is the GMM-UBM paradigm. In this system, speaker-specific GMMs are adapted, via maximum a posteriori (MAP) adaption from a universal background model (UBM), a GMM which models the broad acoustic landscape and is formed using speech samples from a wide range of speakers, reflecting the expected, speakerindependent distribution of feature vectors (Reynolds et al., 2000). The UBM can be viewed as analogous to a rough acoustic clustering, which implicitly performs a soft partitioning of the acoustic space. Work by Kua et al. (2011) shows MFCCs are well suited for performing this soft clustering, providing more stable/reliable partitioning of acoustic space than other spectral features. The effect of MAP-adaption is to capture variations from the broad acoustic landscape defined by the UBM specific to a given set of training data.

In many automatic speaker recognition tasks, it is standard practice to adapt only the means of the Gaussians. The necessary discriminatory information (acoustic variations) required is considered to be in the change of average spectral shapes of acoustic classes for a specific target speaker 
(Reynolds et al., 2000). However, in many speech-based applications, such as language recognition (Campbell, 2008), discriminatory information is considered to also be present in the extent of variations from the GMM mixture means as contained within the GMM covariances. Given the links with decreasing spectral variability we expect this to be the case for speech affected by depression (Cummins et al., 2013a, 2013b).

The advantage to analysing and classifying the effects of depression using the GMM-UBM paradigm, as opposed to a multivariate feature space analysis (Kaya et al., 2014; Low et al., 2011; Valstar et al., 2013), is that it provides a framework for supervector analysis and a subsequent range of transforms designed to help minimize unwanted variability (Campbell et al., 2006; Dehak et al., 2011; Kenny et al., 2008). Provided a suitable amount training data goes into the UBM all acoustic properties of speech should be captured. When adapting with speech data affected by depression MAP captures depression specific acoustic variations from the UBM. However, the acoustic properties captured during MAP depend on all the acoustic factors present in the UBM training data (Reynolds et al., 2000). Therefore MAP will also capture variations from the UBM due to other acoustic factors present in training data. This could include variations due to changing phonetic variability, differing speaker characteristics and differing paralinguistic information.

The successful application of such transforms therefore requires the effects of depression to be the most dominant factor causing the captured variability. Given the results in Cummins et al. (2014a, 2013c, 2011) and Sturim (2011) it is reasonable to assume nuisance factors (variability factors not related to depression) will dilute the depression specific variations in acoustic space. Therefore to find a transform to minimize unwanted variability specific to depression we first need to identify depression-specific variations in acoustic space.

\subsection{Introduction to Measures of Acoustic Variability}

In exploring the hypothesis proposed in Section 1.4; the effects of depression manifest as a reduction in the spread of phonetic events in acoustic space, the focal point of our investigations are 
three measures of acoustic variability; Average Weighted Variance (AWV), Acoustic Movement (AM) and Acoustic Volume measures. Both AWV and Acoustic Volume measures attempt to capture common depression specific variations, from the broad acoustic landscape, as modelled in speakerspecific GMMs whilst AM is estimated from the trajectory of a speech segment in the acoustic landscape (Figure 1).

Given strong links with depression and decreases in spectral energy variability we expect both AWV - an estimate of localized acoustic variability present in a Gaussian mixture model (Section 4.2) - and Acoustic Volume measures - an estimate of the total acoustic variability present in a speech segment (Section 4.4) - to decrease with increasing levels of speaker depression. AM, a marker of spectral variability in an utterance, (Section 4.3), is estimated from the acoustic trajectory of speech segment in acoustic space (Figure 1). Given both a slowing of neuromuscular commands and increasing muscle tension a smoother acoustic trajectory, thus a reduction in AM in speech, should be produced at greater levels of depression.

A more in-depth explanation of all acoustic features and the equations used in their extraction is given in Section 4. This paper, expands on the techniques and results presented in (Cummins et al., 2014b, 2013b); by using two commonly published depression datasets (Section 3) and a wider and more in-depth set of features and statistical analyses, it offers a greater range of insight into the effect of depression in acoustic space. 


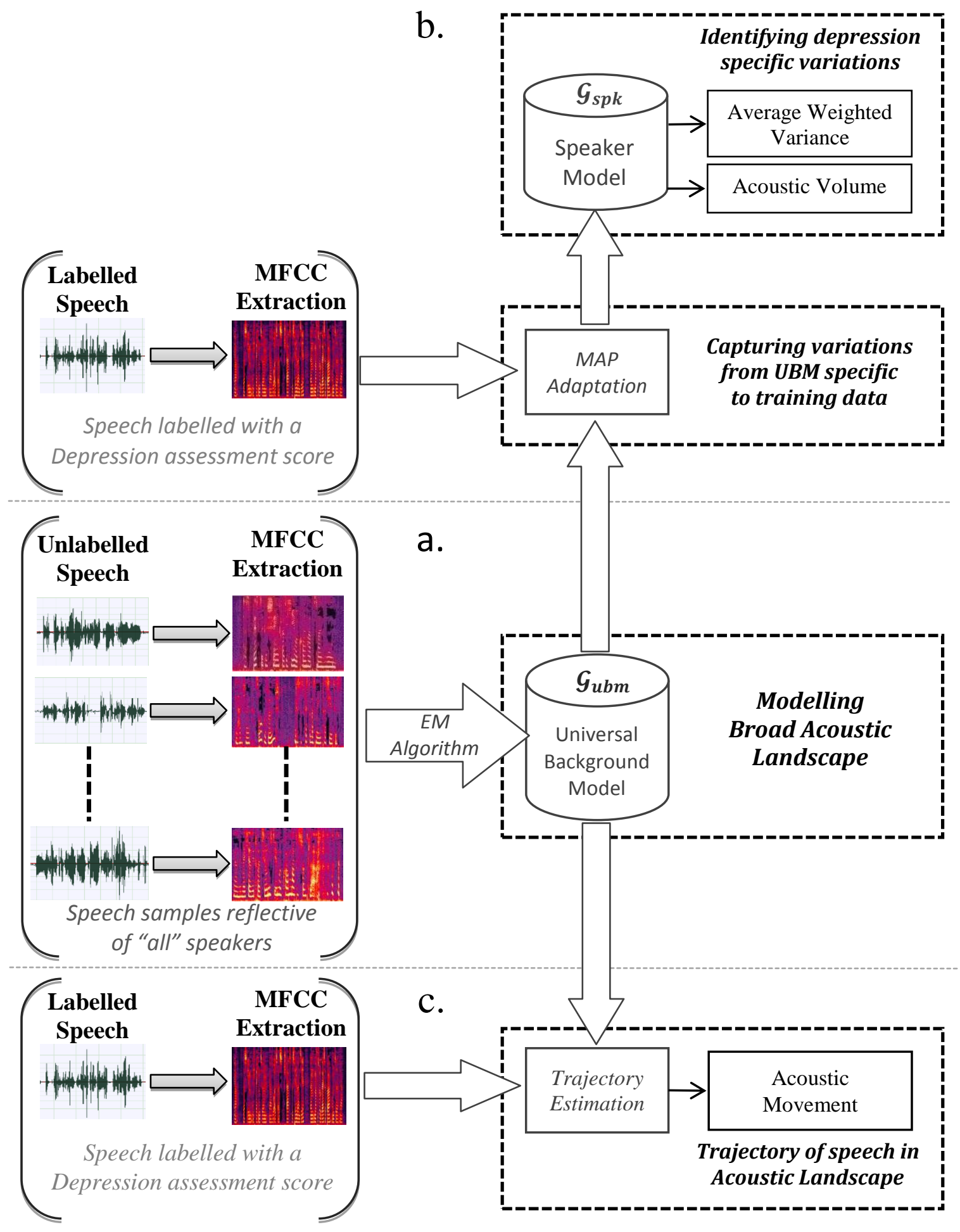

Figure 1: Extraction of Acoustic Space Features (b) extraction of Average Weighted Variance and Acoustic Volume Measures from Speaker Specific Models, (a) formation of a UBM, (c) Extraction of Acoustic Movement from UBM 


\section{Experimental Settings}

\subsection{Depression Corpora}

All depression experimental results in this paper are reported for two commonly published depression datasets. The first is the Audio/Visual Emotion Challenge and Workshop (AVEC) 2013 dataset, herein referred to as AVEC-2013, first detailed in (Valstar et al., 2013). Papers published on this corpus include (Cummins et al., 2014a, 2014b, 2013c; Kaya et al., 2014; Williamson et al., 2013). This corpus consists of 150 files (recordings), taken from the Audio-Visual Depressive Language Corpus, divided into training, development and testing partitions each of 50 files (recordings) with a mean file length of $14 \min 52 \mathrm{sec}$, collected using a headset at a sampling rate of $44.1 \mathrm{KHz}$, with 16 bit encoding (Valstar et al., 2013). All speakers are native German speakers and completed vocal exercises (e.g. held vowels, counting 1 to 10 ) as well as a range of free speech (e.g. speaking out loud whilst solving a task, subject telling a story from their past) and read speech tasks (e.g. Passages from the novel Homo Faber by Max Frisch and the fable Die Sonne und der Nordwind) (Valstar et al., 2013). The AVEC-2013 has data recorded from two different channels (different headset and /or recording equipment), and the different set-ups are denoted by speaker identifiers $200-299$ or 300 and above.

The second corpus is the James Mundt 35-speaker Database, herein referred to as Mundt-35, originally collected for a depression severity study by Mundt et al. (2007). Papers published on this corpus include (Cummins et al., 2013a, 2013b; Helfer et al., 2013; Horwitz et al., 2013; Trevino et al., 2011). Participants in the study undertook fortnightly clinical sessions in which they recorded a variety of speech tasks on a standard office telephone (Sample Frequency $8 \mathrm{kHz}$ ). The recordings are American English and include responses to three questions on the patient's emotional and physical state as well as a reading of the Grandfather Passage a commonly used reading passage used by speech pathologists to test fluency (Wertz et al., 1984). 
All files in both corpora are labelled with self-reported measures of depression; Beck Depression Inventory (BDI) score for AVEC 2013 and the Quick Inventory of Depressive Symptomatology (QIDS) score for Mundt-35. Both assessments are clinically valid self-reported measures able to both differentiate depressed from non-depressed patients and track patient progress during treatment (Maust et al., 2012). The major difference between the two measures is that whilst QIDS assesses each of the nine major symptoms of major depression disorder identified in the DSM-IV, the BDI focuses more on negative self-evaluative symptoms (Maust et al., 2012). A general criticism of selfevaluated diagnostic depression tests is that their reliability can be compromised through patient over familiarity and a patient's reading ability (Cusin et al., 2010).

All results, Tables 3-11, are reported in terms of correlation and 2-class effect size. Correlations are performed to test the significance of possible monotonic relationships between our measures of acoustic variability and depression severity. Whist effect size - defined as the magnitude of the standardized differences in means between two groups (Sullivan and Feinn, 2012) - is used to determine if extracted variability measures can sufficiently differentiate between low and high levels of depression. The evaluation data was formed using the Homo Faber excerpts for AVEC-2013 and the Grandfather Passage for Mundt-35.

All AVEC-2013 correlations are performed on the training and development files denoted by identifiers 200-299; this is done to help minimise the effect of channel variability - there is an audible difference between the two channels the "300+" files have a lower signal-to-noise ratio (SNR) - which has shown to be a confounding factor when classifying level of depression (Sturim et al., 2011). This division results in a total of 68 files from 38 speakers with a BDI range of 0-45 with an average file length of $3 \mathrm{~min} 40 \mathrm{sec}$. All Mundt-35 correlations are performed on the in-clinic recordings - minimising the effect of channel - from the Caucasian patients to help minimise the effect of variability due to accent. Noting also that not every speaker participated in every clinical session; this resulted in 118 recordings, with an average length of 50s, spread over 32 speakers. 
For the effect-size tests both the AVEC-2013 and Mundt-35 were arranged into two speaker independent groups ${ }^{1}$. The AVEC-2013 training and development files with identifiers 200-299 were divided into two partitions of 26 files. The first, denoted $A_{-}$Low, contains files with a BDI $\leq 12$, whilst the second, denoted $A_{-}$High, contains files from a separate set of speakers, with a BDI $\geq 20$. Similarly the Mundt-35 files were divided into two partitions of 43 files. The first, denoted M_Low, contains files with a QIDS $\leq 10$, whilst the second, denoted $M_{-}$High, contains files from a separate set of speakers, with a QIDS $\geq 16$. The authors would like to highlight that 2-class data division was done before generating results and the speaker independent set-up was a deliberate decision to further help maximise our chances of identifying acoustic changes relating to depression, since speaker identity is also a confounding factor in when classifying depression (Cummins et al., 2014a, 2011).

It is worth noting limitations of both corpora which include the effects of age, medication and channel. Both AVEC-2013 and Mundt-35 have large age ranges; 45 and 48 years respectively. The MFCC-GMM paradigm can be used to identify age, albeit with limited accuracy (50-55\%, 4-class classification problem (Schuller et al., 2013)). There is a range of antidepressant medications present in the Mundt-35 corpus whilst there are no medication records for speakers in AVEC-2013. Antidepressants have possible influences on the speaker's voice. Common side effects which can alter speech production include; dry mouth (inadequate saliva production), fatigue, tremors and agitation (Crawford et al., 2014; Kikuchi et al., 2012). A series of preliminary experiments was done on the AVEC-2013 which established a significant differences in acoustic properties between the "200" identifier files and " 300 " identifier files. The results of this preliminary analysis - not given - could be best explained by the difference in channel between the two file groups. Therefore we took the decision to remove the " 300 " identifier files from our analysis.

\footnotetext{
${ }^{1}$ Speaker groupings available on request
} 


\subsection{Key Settings}

For Section 4 the experimental settings, unless otherwise stated, were as follows: frame level features comprised 13 MFCCs, including C0, appended with delta $(\Delta)$ and delta-delta $(\Delta \Delta)$ coefficients, which were extracted according to (Cummins et al., 2013c) for the AVEC-2013 and according to (Cummins et al., 2013b) for the Mundt-35 databases using openSMILE (Eyben et al., 2010). Only voiced frames were used in the modelling, determined using openSMILE's voicing probability function. All individual speaker GMM's were formed using the GMM-UBM paradigm (Figure 1). All UBMs were trained with 10 iterations of the EM algorithm and as per (Cummins et al., 2013b, 2013c) all speaker specific GMM's were formed using full adaption, with five iterations of the MAP algorithm. The AVEC-2013 UBM's were formed using the full testing partition, approximately 13hrs of data, while for the Mundt-35 data the UBM's were formed using the free-response answers, 320mins of training data. All GMM training was done using HTK.

Due to the ordinal / ranked nature of depression scores (Faries et al., 2000), all correlation results are reported in terms of Spearman's rank coefficient $\left(r_{s}\right)$ and $p$-value $(p)$ (Myers et al., 2010). We interpret the $p$-values in the following manner; $p \geq .05$ indicates result is not significant, $p<.05$ indicates result is significant, $p<.01$ indicates significance at a moderate level and $p<.001$ indicates significance at a strong level. Effect size - the magnitude of the differences in means between two groups - is calculated using Hedge's G coefficient $(g)$ and interpreted in the following manner; effect size of $g \pm 0.2$ is a mild effect, $g \pm 0.5$ is a moderate effect and $g \pm 0.8$ is a strong effect (Rosenthal, 1994). Further significance for the effect size is provided for the effect size in terms of the $p$-value found using two-class t-tests; these p-values are interpreted as above. Significance, as indicated by a $p$-value, is the probability the difference between the two groups could just due to chance and can be confounded by sample size. Effect size is the quantification of size of the differences between two data groupings regardless of sample size. We use effect size as an indicator of how effective are our 
variability measures in differentiating between low and high levels of depression - small effect sizes are unlikely to have much utility for classification tasks.

\section{Features and Statistical Analysis}

\subsection{Feature Space Mean and Variance}

For a lack of acoustic variance, due to depression, to be observable as modified acoustic model parameters it is reasonable to assume there first needs to be a reduction in spectral variations present in the (frame-level) feature domain. A consistent trend of negative correlations in sub-band energy variability with increasing levels of depression has been previously reported for the Mundt35 corpus, (Cummins et al., 2013a, 2013b), similar results for the AVEC-2013 data are shown below. In terms of consistency in both the size and magnitudes of the correlation coefficients; the mean of each MFCC feature dimension is not well correlated for AVEC-2013, Figure 2(a). However, the variance of each MFCC feature dimension, especially the $\Delta$ and $\Delta \Delta$ coefficients are strongly negatively correlated with depression severity Figure 2(b). This result is consistent with Mundt-35 results, Figure 2(c) and Figure 2(d) - reproduced from (Cummins et al., 2013b). 

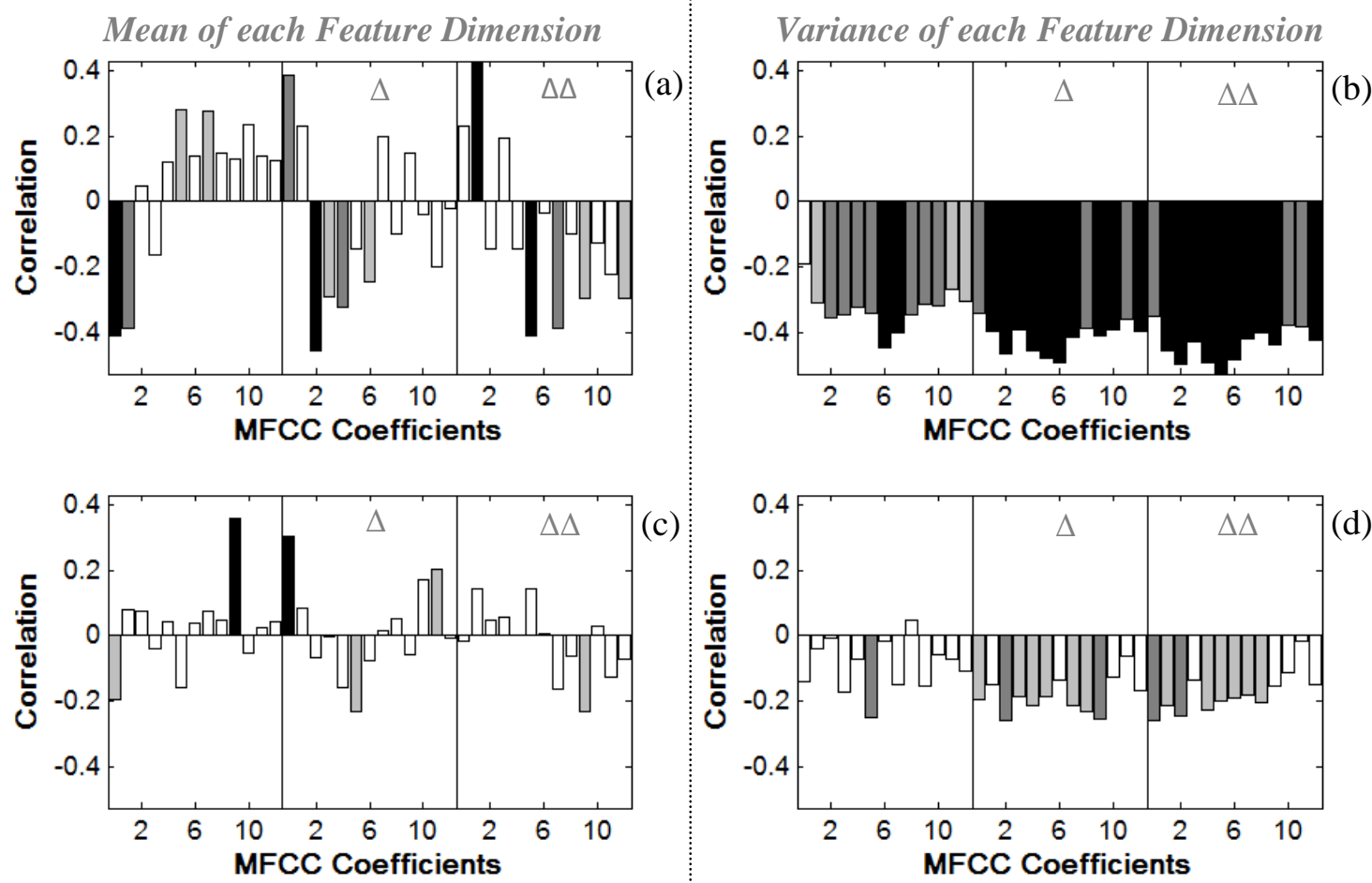

Figure 2: Correlations per feature dimension, calculated on AVEC-2013, for the mean (a) and variance

(b) for MFCCs (appended with delta coefficients) with BDI scores. For comparison the correlations per feature dimension, calculated on Mundt-35, for the mean (c) and variance (d) for MFCCs (appended with delta coefficients) with QIDS scores have been reproduced from (Cummins et al., 2013b). Light-Grey indicates significance $(p<.05)$, Dark-Grey indicates moderate significance $(p<.01)$ and black indicates strong significance $(p<.001)$. Correlations were found with $n=68$ files for AVEC-2013 and $n=118$ files for Mundt-35

\subsection{Average Weighted Variance}

Average Weighted Variance (AWV) is a simple estimator of the average local variability captured by a GMM (Figure 3) first proposed by Hasan and Hansen (2011) for measuring variability in a UBM. AWV is computed using the weights and diagonal covariance matrices of a given GMM:

$$
A W V=\frac{1}{K} \sum_{i=1}^{M} \sum_{j=1}^{K} \omega_{i} \sigma_{i, j}^{2}
$$


where $M$ is the number of mixtures, $K$ the feature space dimensionality, and $\sigma_{i, j}^{2}$ is the $\mathrm{j}$-th diagonal covariance component taken from the i-th mixture. Note that unless otherwise stated, the results in this section were found using variance and weight (vw) MAP adaption.

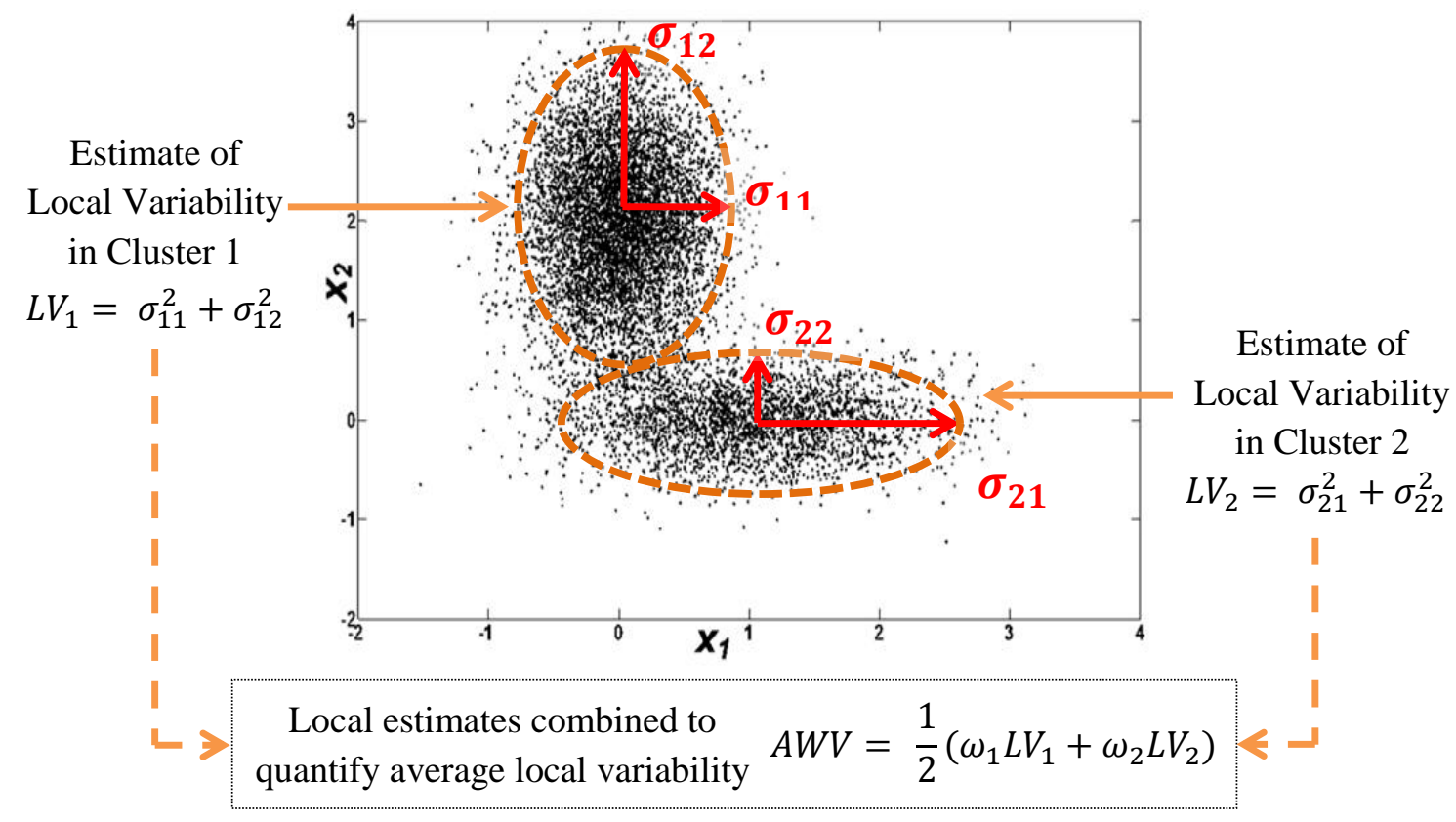

Figure 3: An example of a synthetic 2D feature space $\left(x_{1}, x_{2}\right.$, arbitrary 'feature values' for illustrative purposes) with two main feature clusters and the local variability (LV) estimates used to calculate Average Weighted Variance

Given strong links between depression and decreases in spectral energy variability (refer Section 4.1) and the similar effects of depression on phone-spread for phonemes grouped together by manner of articulation (Trevino et al., 2011), we expect there will be a decrease in localised variability in acoustic space which will result in an overall decrease in AWV. Strong evidence for this decrease is seen in the AVEC-2013 dataset Table 3, where strong correlations $(p<.001)$ and moderate effect size results $(|g|>0.5, p<.01)$ were found across all mixtures. The results for the Mundt-35 corpus are not as strong as for AVEC-2013, significant correlations are seen for the 8-, 32- and 128-mix GMM's and only mild effect size $(0.2<|g|<0.5)$ is seen across all mixes (Table 3 ). The differences in AWV results between the two corpora are discussed further in Section 5 . All statistical tests presented in Table 3 match the results presented in Cummins et al. (2013b), indicating a decrease in AWV with increasing 
levels of depression, providing evidence that the average local variability captured by a GMM deceases with increasing levels of speaker depression².

Table 3: Statistical test results for AWV calculated for both the AVEC-2013 and Mundt 35 Speaker Depression Corpora. Correlations were found with $n=68$ files for AVEC-2013 and $n=118$ files for Mundt-

35. Effect size results were found using $n=26$ files per class for AVEC-2013 and $n=43$ files per class for Mundt-35.

\begin{tabular}{|c|c|c|c|}
\hline Corpus & $\begin{array}{l}\text { GMM } \\
\text { Mix }\end{array}$ & $\begin{array}{c}\text { Correlation } \\
r_{s} \\
\end{array}$ & $\begin{array}{c}\text { Effect Size } \\
g\end{array}$ \\
\hline \multirow{5}{*}{ AVEC-2013 } & 8 & $-0.42 * * *$ & $0.68^{*}$ \\
\hline & 16 & $-0.43 * * *$ & $0.68 *$ \\
\hline & 32 & $-0.44 * * *$ & $0.69 *$ \\
\hline & 64 & $-0.44 * * *$ & $0.70^{*}$ \\
\hline & 128 & $-0.44 * * *$ & $0.72 *$ \\
\hline \multirow{5}{*}{ Mundt-35 } & 8 & $-0.19 *$ & 0.31 \\
\hline & 16 & -0.15 & 0.29 \\
\hline & 32 & $-0.18 *$ & 0.34 \\
\hline & 64 & -0.17 & 0.30 \\
\hline & 128 & $-0.18^{*}$ & 0.30 \\
\hline
\end{tabular}

As in Cummins et al. (2013b) all tests presented in Table 3 were found using variance and weight only (vw) MAP adaptation. As we are interested in variations in acoustic space, captured in a GMM's covariance and weighting parameters ${ }^{3}$, this approach seems reasonable. Performing full adaption on AVEC-2013 results in minimal change between the statistical analysis results when compared to vw adaptation (Table 4). Whilst for when performing full adaption on Mundt-35 there is little change in correlation but a substantial drop in effect-size when compared to vw adaptation (Table 4).

For both date sets we see a drop in the class (level of depression) mean AWV values when comparing full adaptation to vw adaption (Table 4). Calculating the per class effect size between the adaption methods shows that this drop in AWV is strongly significant (Table 4). Note that this change

\footnotetext{
${ }^{2}$ The Mundt results in this section differ from those presented in Cummins et al. (2013b) as they are obtained using a different UBM. In Cummins et al. (2013b) the UBM was formed using the NIST 2004 Speaker Recognition Evaluation dataset

${ }^{3}$ Weights control the mixture occupancy count (the amount of data assigned to a mixture during MAP adaptation) capturing spectral variability on a global level as opposed to the localised variability captured in the covariances
} 
in AWV with adaption method is seen across both corpora. We therefore speculate that the change in means during adaptation accounts for a proportion of the feature space variance resulting in inconsistencies when comparing acoustic variations between speakers.

Table 4: Differences between full and vw adaptation on 32 mix GMM AVW results for both corpora. Correlations were found with $n=68$ files for AVEC-2013 and $n=118$ files for Mundt-35. Effect size results were found using $n=26$ files per class for AVEC-2013 and $n=43$ files per class for Mundt-35.

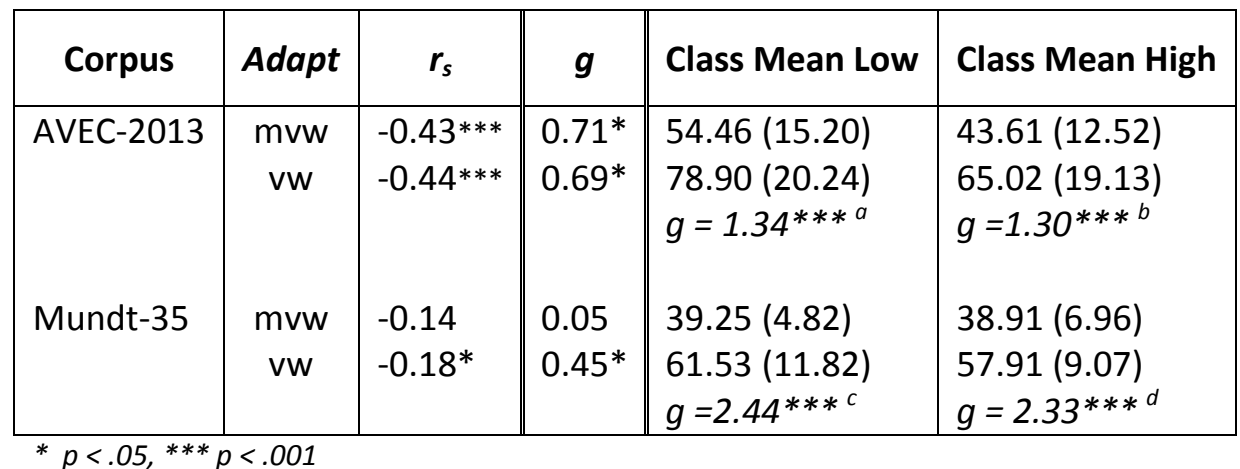

a) - Effect Size betweenAVEC-2013 AWV vw and mvw Low class results

b) - Effect Size between AVEC-2013 AWV vw and mvw High class results

c) - Effect Size betweenMundt-35 AWV vw and mvw Low class results

d) - Effect Size between Mundt-35 AWV vw and mvw High class results

Viewing the UBM as a soft partitioning of the acoustic space (Section 2), vw adaptation ensures there is no loss of correspondence between mixtures during MAP adaptation restricting the acoustic variations captured during MAP adaptation to be due to differences in acoustic sounds as defined by the UBM. Allowing means to adapt potentially redefines the UBM's acoustic partitioning for each speaker. It is this redefinition that somehow accounts for a proportion of the feature space variance, reducing the efficiency of $A W V$ as a marker of depression. Further evidence for this is provided in Figure 4 where a significant drop in AWV can be seen, for both the high and low depression classes in the AVEC-2013 corpus, when comparing full adaptation versus the vw adaptation across successive MAP iterations. Tests to quantify exactly how shifting means reduces AWV have so far proved unsuccessful and will be a focus of future research. We note that whilst the results in Table 4 and Figure 4 are for 32 mix UBM similar results were seen across all UBM sizes. 


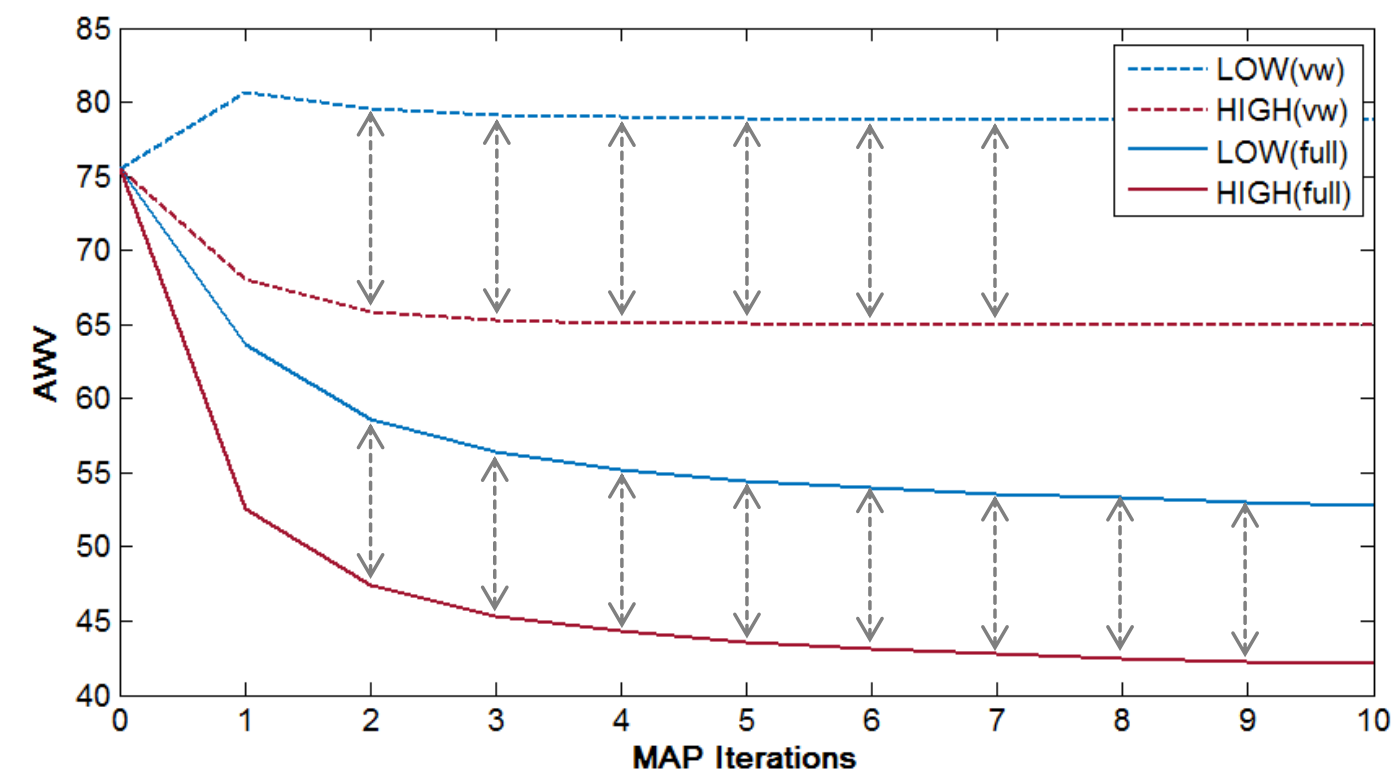

Figure 4: Comparing Average Weighted Variance found using either full or variance and weight only MAP adaption for High and Low levels of depression for a the AVEC-2013 data using a 32 mix GMM

\subsection{Acoustic Movement}

Acoustic Movement (AM) is a marker of spectral variability estimated from the trajectory of an utterance in the acoustic landscape. It was originally proposed by Krishnamurthy and Hansen (2009) to analyse the acoustic trajectory of multi-speaker babble to determine if an audio segment contained speech from a single speaker or multiple speakers. The acoustic trajectory of speech from a single subject is expected to be smoother than multi-speaker babble; the inertial nature of speech production does not allow recurring abrupt acoustic movements. We note that AM is not analogous to speech rate; it is a marker of the smoothness of the acoustic trajectory of an utterance or the average amount of movement between (quantised) acoustic regions in an utterance as opposed to a marker of units of sound per second.

To calculate AM for a given set of frame level speech features $X=\left\{\boldsymbol{x}_{1} \cdots \boldsymbol{x}_{T}\right\}$, we first need to calculate $d$ an indicator of movement between the individual mixture components, of a given GMM (Krishnamurthy and Hansen, 2009). Given $X$ and a corresponding GMM-UBM, the posterior probability of each mixture given each frame in $X$ is calculated and the mixture corresponding to the 
highest posterior probability is recorded. $d$ is then a binary marker of acoustic movement; returning a 1 when consecutive frames in $X$ coincide with different mixture components and returning a 0 otherwise:

$$
d(i)=\left\{\begin{array}{cc}
1, & \arg \max _{i} P\left(\gamma_{i} \mid \boldsymbol{x}_{t}\right) \neq \arg \max _{i} P\left(\gamma_{i} \mid \boldsymbol{x}_{t-1}\right) \\
0, & \text { otherwise }
\end{array}\right.
$$

where

$$
P\left(\gamma_{i} \mid \boldsymbol{x}_{t}\right)=\frac{\omega_{i} \mathcal{N}\left(\boldsymbol{x}_{t} ; \boldsymbol{\mu}_{i}, \sum_{i}\right)}{\sum_{j=1}^{M} \omega_{j} \mathcal{N}\left(\boldsymbol{x}_{t} ; \boldsymbol{\mu}_{\boldsymbol{j}}, \sum_{j}\right)}
$$

where $\gamma$ represents an individual mixture component in a M-mix GMM-UBM. The total number of 1 's given by $d$ is the total number of acoustic 'hops' per utterance; the larger this number the greater the amount of spectral variation in $X$. For the purpose of this paper Acoustic Movement is defined as the mean number hops per utterance:

$$
\mathrm{AM}=\frac{\text { total number of hops }}{\text { Number of frames in utterance }}
$$

If the value of $A M$ is 1 , the implication is that every frame in the utterance corresponds to a different mixture component in $\mathcal{G}$ when compared to the previous frame (Krishnamurthy and Hansen, 2009). Note that no VAD was used when generating the AM results.

In the literature there is evidence for a slowing of articulatory muscles with increasing level of speaker depression (Flint et al., 1993; Mundt et al., 2012). Given this effect and the decrease in spectral variability, Section 4.1, we expect a smoother acoustic trajectory - thus a reduction in AM in speech affected by depression.

The AM results for both AVEC-2013 and Mundt-35 are not consistent across UBM sizes (Table 5). For AVEC-2013 only 16-mix and 64-mix UBM provide significant correlations and effect sizes (Table 5). 
Whilst for Mundt-35 only 8-mixUBM provides both a significant correlation and effect size (Table 5). The differences in AM results between the two corpora are discussed further in Section 5.

Table 5: Statistical Analysis results for Acoustic Movement calculated for both the AVEC 2013 and Mundt 35 Speaker Depression Corpora. Note: no VAD was used in these calculations. Correlations were found with $n=68$ files for AVEC-2013 and $n=118$ files for Mundt-35. Effect size results were found using $n=26$ files per class for AVEC-2013 and n=43 files per class for Mundt-35.

\begin{tabular}{|c|c|l||l|}
\hline Corpus & $\begin{array}{c}\text { GMM } \\
\text { Mix }\end{array}$ & $\begin{array}{c}\text { Correlation } \\
r_{s}\end{array}$ & $\begin{array}{c}\text { Effect Size } \\
g\end{array}$ \\
\hline & 8 & 0.11 & -0.19 \\
& 16 & $-0.25^{*}$ & $0.61^{*}$ \\
AVEC-2013 & 32 & -0.23 & 0.52 \\
& 64 & $-0.26^{*}$ & 0.52 \\
& 128 & -0.15 & 0.25 \\
& & & \\
& 8 & $-0.23^{*}$ & $0.53^{*}$ \\
Mundt-35 & 16 & $-0.19^{*}$ & 0.27 \\
& 32 & -0.09 & -0.01 \\
& 64 & 0.01 & -0.30 \\
& 128 & -0.01 & -0.24 \\
\hline$p<.05$
\end{tabular}

The Mundt-35 AM results for UBM sizes $M>16$, are inconsistent, in terms of size and direction of the statistical effects, with those reported for both the lower UBM sizes, M $\leq 16$, and AVEC-2013 results. It is possible that the UBM's, $M=128$ (AVEC-2013) and $M \geq 32$ (Mundt-35), are an unsuitable partitioning of the acoustic space for their corpora. This could potentially be due to two reasons (i) insufficient acoustic variability in the test utterance for the larger UBM sizes, or (ii) insufficient acoustic variability in UBM training data resulting in a sparse clustering of the acoustic space during UBM training.

The AVEC-2013 $(M=16, M=64)$ and Mundt-35 $(M \leq 16)$ results provide evidence that the acoustic trajectory of highly depressed speech is smoother than speech produced at low levels of depression. From this we infer that with increasing levels of speaker depression there is less distinction between distinct phonetic sounds. This matches nicely slurred descriptors of depressed voice (Helfer et al., 2013; Williamson et al., 2013) and complements the results by Trevino et al. (2011) who report 
similarities in average phonetic durations when grouping phonemes together by manner of articulation.

When calculating AM we also recorded the number of times each specific mixture component returned the maximum posterior probability. For the AVEC-2013 dataset (results not given) there was a statistically significant reduction in the total number of mixture components associated with the maximum posterior probability. We interpret this result to indicate that - as well as a smoother acoustic trajectory - with increasing levels of speaker depression a speaker's trajectory covers a reduced area of acoustic space. This provides further evidence for a reduction in global spectral variability with increasing levels of speaker depression in this corpus.

\subsection{Acoustic Volume}

For the purpose of this paper, we use the term Acoustic Volume to notionally refer to the size of the feature space occupied by feature vectors extracted from some speech data (Figure 5). We expected, given results in (Cummins et al., 2014b; Krishnamurthy and Hansen, 2009), that the relative size of this region reflects acoustic variability and consequently varies between speakers due to speaker characteristics, phonetic content and paralinguistic phenomena modulated into the speech utterance/segment. We expect that due to a lack of speech motor control, Section 2, there is a reduction in acoustic variation - thus a reduction in Acoustic Volume - in speech produced under greater levels of depression. In this Section we introduce three measures for estimating volume; GMM Mean Acoustic Volume (Section 4.4.1); Inter-Centroid Distance (Section 4.4.2) and the novel Probabilistic Acoustic Volume (Section 4.4.3) method. For each measure we provide their respective experimental results and analysis. 


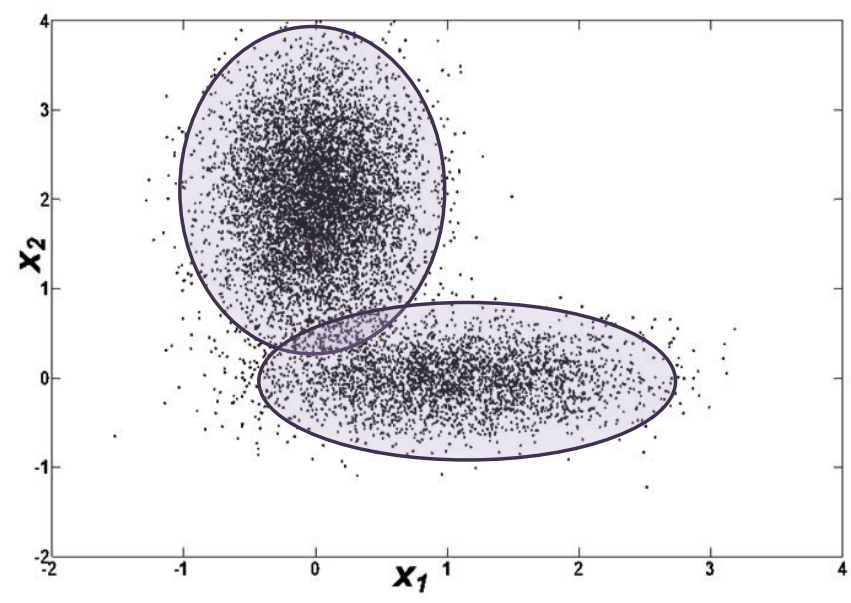

Figure 5: An example of a synthetic 2D feature space $\left(x_{1}, x_{2}\right.$, arbitrary 'feature values' for illustrative purposes) with two main feature clusters. The shaded area represents an estimate of the Acoustic Volume of this feature space. The measures discussed in Section 4 represent different methods to estimate this volume

\subsubsection{GMM Mean Acoustic Volume}

Krishnamurthy and Hansen (2009) first proposed the GMM Mean Acoustic Volume (GM-AV) technique for estimating acoustic variation in babble noise. This method uses a set of GMM means to define a hyper cuboid, Figure 6, the volume of which is an indicator of the acoustic variation present in the data. For the complete description of the technique see (Krishnamurthy and Hansen, 2009).

Given an $M$-mixture GMM which adequately describes the distribution of $\mathrm{N}$-dimensional features extracted from a speech segment, Krishnamurthy and Hansen (2009) states there exists an Ndimensional hyper-cuboid of minimal volume that contains $\mathbb{M}$, the set of mixture means $\mathbb{M}=$ $\left\{\boldsymbol{\mu}_{1}, \cdots, \boldsymbol{\mu}_{M}\right\}$, within it. This cuboid has $2^{N}$ vertices characterized by the set of maxima and minima points evaluated for each of the $N$ dimensions separately across all vectors in $\mathbb{M}$. It is possible to estimate the volume enclosed by this set by identifying the set of $N$ edge lengths $\left\{e_{1}, e_{2}, \ldots, e_{N}\right\}$ which enclose the cuboid. Each edge $e_{k}$ is defined by the maxima and minima points of the $k^{\text {th }}$ feature dimension: 


$$
e_{k}=\max \left(n_{k}\right)-\min \left(n_{k}\right)
$$

where $n_{k}=\left\{\mu_{1}^{(k)}, \mu_{2}^{(k)}, \ldots, \mu_{M}^{(k)}\right\}$ is a set of $M$ elements and $\mu_{i}^{(k)}$ denotes the $k^{t h}$ dimension of the $i^{\text {th }}$ mean vector, $\boldsymbol{\mu}_{i} \in \mathbb{M}$. The volume of cuboid (GM-AV) is then given by:

$$
V=e_{1} \times e_{2} \times \ldots \times e_{N}
$$

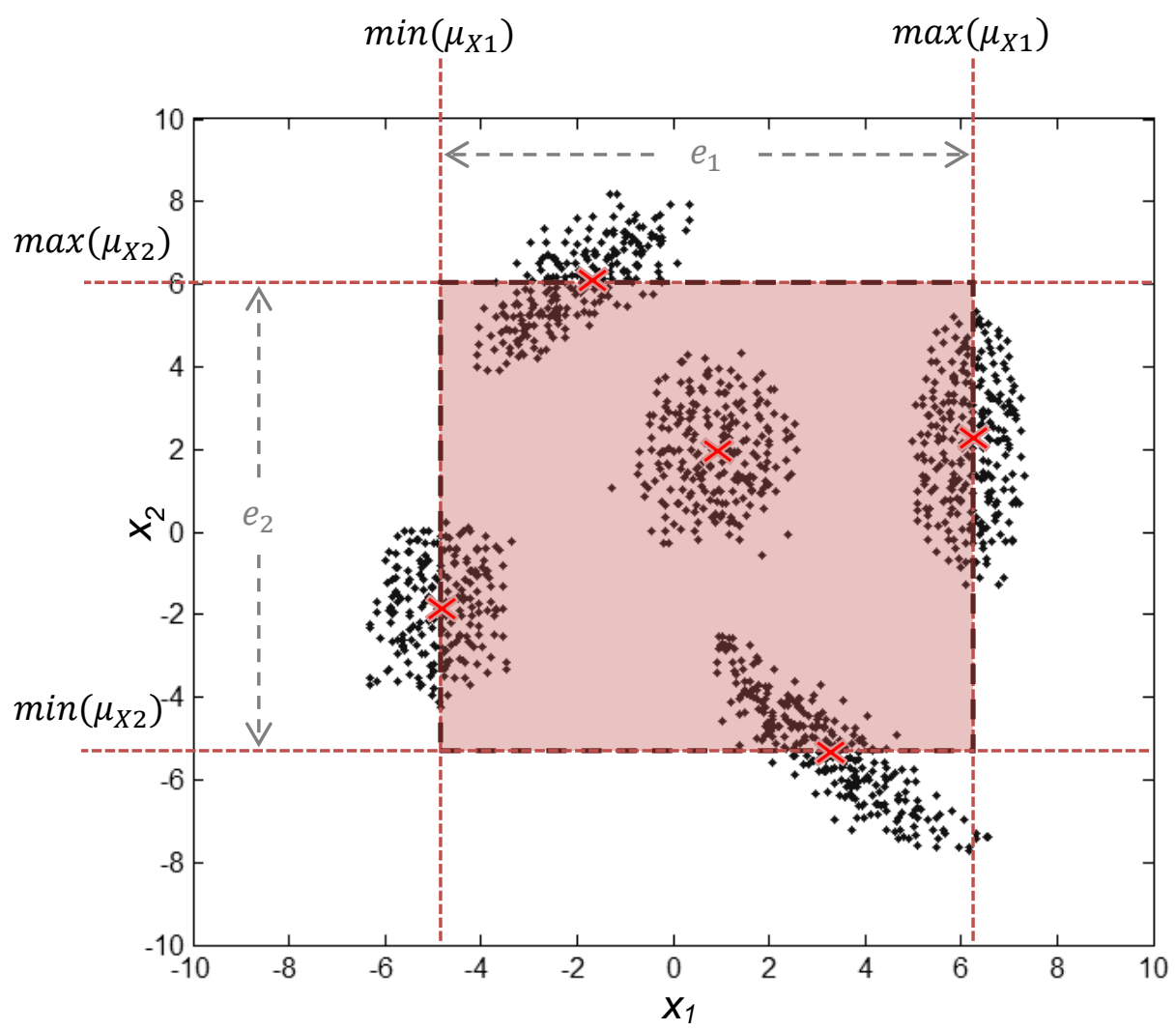

Figure 6: An example of a synthetic $2 D$ feature space $\left(x_{1}, x_{2}\right.$, arbitrary 'feature values' for illustrative purposes) with five main feature clusters. The red crosses indicate the cluster means and the shaded area $e_{1} \times e_{2}$ represents an estimate of the GM-AV of this feature space.

As GM-AV is proportional to the amount of acoustic variation present in some speech data (Krishnamurthy and Hansen, 2009), which we expect will decrease with increasing levels of speaker depression. The negative correlations for both corpora, Table 6, provide evidence that indeed GM- 
AV decreases with increasing levels of depression ${ }^{4}$. For AVEC-2013, significant correlations $(p<.05)$ are seen for 8-, 16-, and 32-mixes and moderate correlations $(p<.01)$ for 64-, and 128-mixes, whilst for Mundt-35 mild correlations $(p<.05)$ are seen for $16-, 32$ - and 64-mixes. Surprisingly, given the strong correlations, the effect size results $(|g| \leq 0.5, p \geq .05)$ for both corpora are disappointing indicating GM-AV cannot sufficiently differentiate between low/high levels of depression.

Table 6: Statistical Analysis and Score Level Prediction Results for GM-AV, calculated for both the AVEC2013 and Mundt 35 Speaker Depression Corpora. Correlations were found with $n=68$ files for AVEC-2013 and $n=118$ files for Mundt-35. Effect size results were found using $n=26$ files per class for AVEC-2013 and $n=43$ files per class for Mundt -35 .

\begin{tabular}{|c|c|c|c|}
\hline Corpus & $\begin{array}{l}\text { GMM } \\
\text { Mix }\end{array}$ & $\begin{array}{c}\text { Correlation } \\
r_{s} \\
\end{array}$ & $\begin{array}{c}\text { Effect Size } \\
g\end{array}$ \\
\hline \multirow{5}{*}{ AVEC-2013 } & 8 & $-0.26^{*}$ & 0.28 \\
\hline & 16 & $-0.26 *$ & -0.11 \\
\hline & 32 & $-0.28^{*}$ & 0.26 \\
\hline & 64 & $-0.32^{* *}$ & 0.28 \\
\hline & 128 & $-0.36^{* *}$ & 0.48 \\
\hline \multirow{5}{*}{ Mundt-35 } & 8 & -0.12 & 0.30 \\
\hline & 16 & $-0.20 *$ & 0.26 \\
\hline & 32 & $-0.23^{*}$ & 0.30 \\
\hline & 64 & $-0.20 *$ & 0.16 \\
\hline & 128 & -0.15 & 0.09 \\
\hline
\end{tabular}

The inconsistences between the statistical results can be explained by the GM-AV boxplot (Figure 7). The outliers in both classes are considerably larger than the main body of GM-AV values; the $y$-axis is in log scale to allow the boxes to be visualized. The outliers, as well as having a strong effect on the location of the mean of each class, would also (given the larger numbers of outliers in the low class), have an undue effect on the correlation calculations. By removing these outliers it is possible to find mildly significant differences $(|g| \geq 0.5, p<.05)$ between the two classes, nevertheless as removing

\footnotetext{
${ }^{4}$ The GM-AV results in this section differ from those presented in (Cummins et al., 2014b), as they are obtained using on the AVEC-2013 files with an identifier between 200 and 299, Further they are also obtained over a greater number of GMM mixes and include the Mundt-35 results.
} 
outliers is a heuristic process the GM-AV feature appears not suitable for robustly identifying depressed individuals.
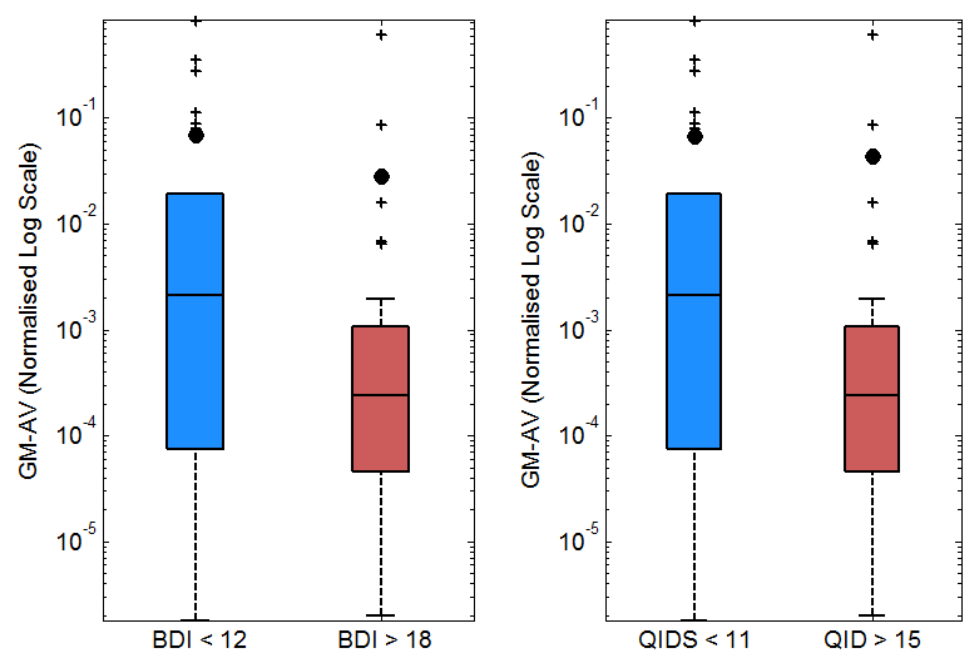

Figure 7: Distribution of GM-AV for AVEC2013 (Left) and Mundt-35 (Right) for both the low and high level of depression classes, found using a 32 mix and 8 mix GMM respectively. Black dots show the location of the mean of each class. Note: $n=26$ files per class for AVEC-2013 and $n=43$ files per class for Mundt-35.

\subsubsection{Inter-Centroid Distance}

Krishnamurthy and Hansen (2009), also proposed the Inter-Centroid Distance (ICdist) as an estimate of acoustic variation. ICdist is the average Euclidean distance between all pairs of means in $\mathbb{M}$, the set of mixture means (Figure 8). This is a marker of acoustic space spread: as acoustic variation decreases the mean vectors move closer together. Where GM-AV is an estimate of the minimum volume of the hyper-cuboid defined by $\mathbb{M}$, ICdist is the arithmetic mean of the distances between elements of $\mathbb{M}$. 


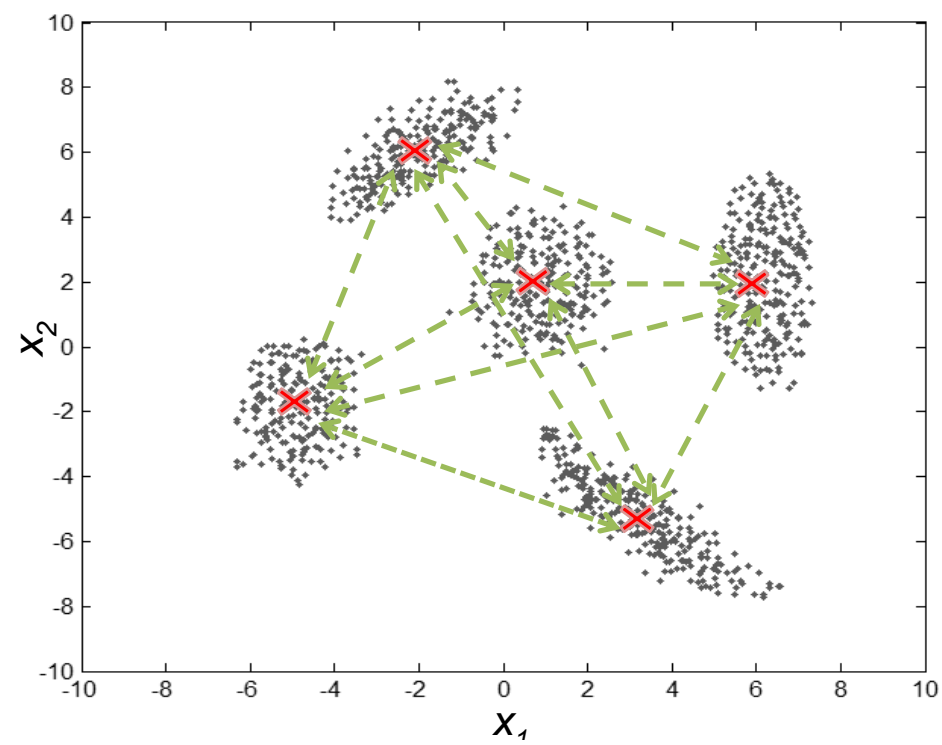

Figure 8: An example of a synthetic $2 D$ feature space $\left(x_{1}, x_{2}\right.$, arbitrary 'feature values' for illustrative purposes) with five main feature clusters. The red crosses indicate the cluster means and the green dashed arrows represent the distances used to calculate the Inter-Centroid Distance

As ICdist is a global estimate of acoustic volume providing information on the closeness of the cluster (phonetic events) in $\mathbb{M}$, in acoustic space, we expect it will also decrease with increasing levels of speaker depression. The negative correlations, for both corpora, show that as expected ICdist decreases with increasing levels of depression (Table 7). For AVEC-2013, similar to the GM-AV measure, the effect size results show that ICdist struggles to differentiate between low/high levels of depression in this corpus. For Mundt-35 the strong and consistent correlation $(p<.01)$ and the effect size results $(|\mathrm{g}| \geq 0.5, \mathrm{p}<.05)$ show ICdist is a good measure for capturing lack of acoustic spread in this corpus. Horwitz et al. (2013) found on Mundt-35 that the variance of the 1st to 3rd formant was significantly negatively correlated with depression, consistent with a slurred voice quality. This can explain, in part, why the ICdist measure works well with Mundt-35, with increased slurring therefore the ability to distinguish between phoneme spectra decreases potentially moving GMM mean vectors closer together in turn decreasing ICdist. 
Table 7: Statistical Analysis for ICdist, calculated for both the AVEC 2013 and Mundt 35 Speaker Depression Corpora. Correlations were found with $n=68$ files for AVEC-2013 and $n=118$ files for Mundt35. Effect size results were found using $n=26$ files per class for AVEC-2013 and $n=43$ files per class for Mundt-35.

\begin{tabular}{|c|c|l||l|}
\hline Corpus & $\begin{array}{c}\text { GMM } \\
\text { Mix }\end{array}$ & $\begin{array}{c}\text { Correlation } \\
r_{s}\end{array}$ & $\begin{array}{c}\text { Effect Size } \\
g\end{array}$ \\
\hline & 8 & $-0.31^{* *}$ & 0.41 \\
& 16 & $-0.32^{* *}$ & 0.50 \\
AVEC-2013 & 32 & $-0.27^{*}$ & 0.43 \\
& 64 & -0.16 & 0.29 \\
& 128 & $-0.25^{*}$ & 0.47 \\
& & & \\
& 8 & $-0.26^{* *}$ & $0.54^{*}$ \\
Mundt-35 & 16 & $-0.25^{* *}$ & $0.61^{*}$ \\
& 32 & $-0.28^{* *}$ & $0.53^{*}$ \\
& 64 & $-0.26^{* *}$ & $0.50^{*}$ \\
& 128 & $-0.23^{*}$ & $0.46^{*}$ \\
\hline
\end{tabular}

\subsubsection{Probabilistic Acoustic Volume Profile}

Neither the GM-AV nor the ICdist calculation takes into account the weight of each component of the GMM when calculating an edge. This makes them susceptible to potentially significant overestimation of acoustic volume if low-weight mixtures with means far from feature clusters are present as illustrated in Figure 9. Specifically, in the example shown, the difference between the extremely low weight mixture 3 being centred at $X=8$ (Figure 9a)and being centred at $X=$ 3 (Figure $9 b$ ) is negligible when the overall probability density modelled by the GMM is considered, however the difference in acoustic volume, estimated using either GM-AV or ICdist, is significant. 

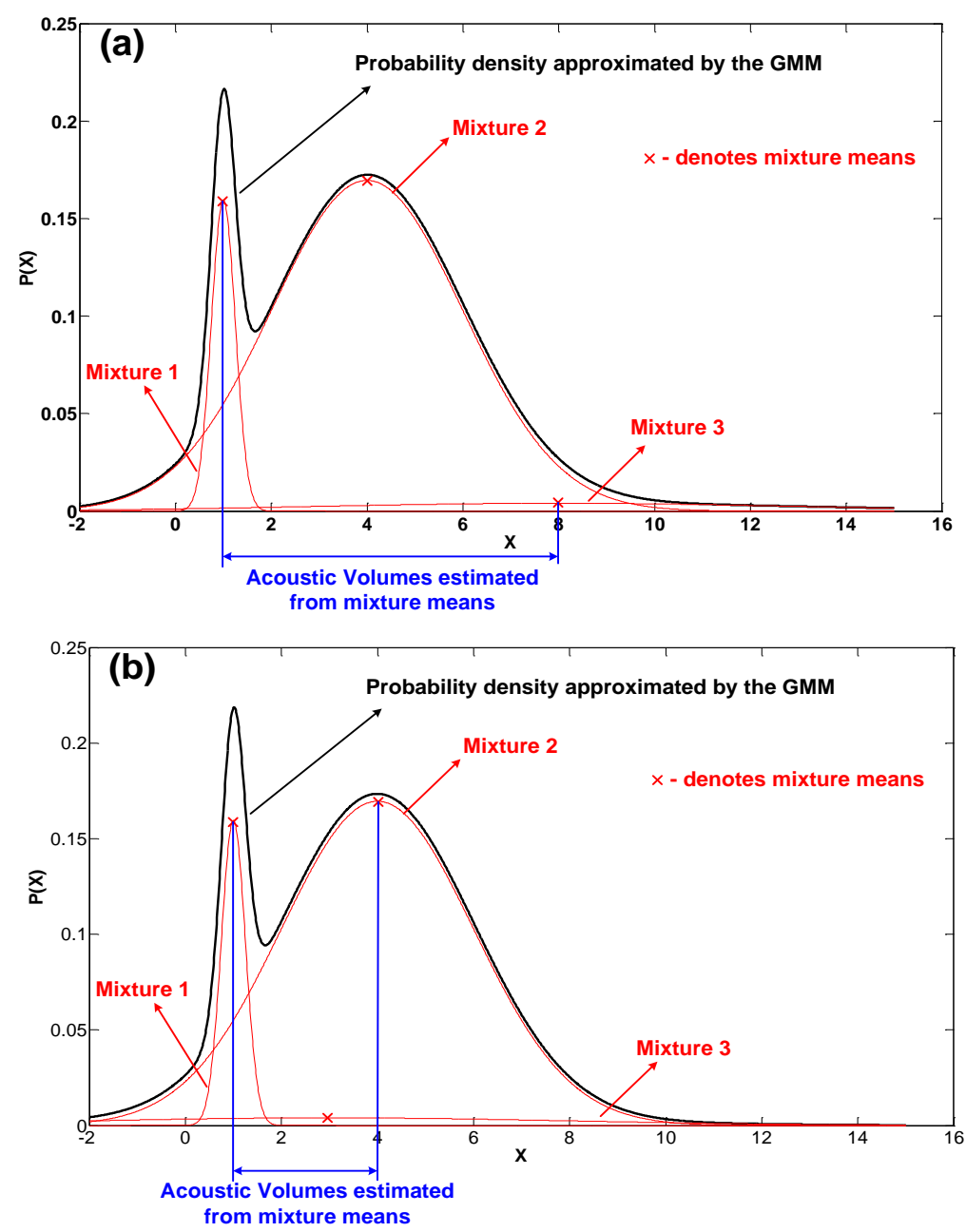

Figure 9: (a) 1-dimensional 3-mixture GMM with low weight mixture 3 centered at $X=8$; (b) 1-

dimensional 3-mixture GMM with low weight mixture 3 centered at $X=3$

An alternative and potentially more robust estimate of acoustic volume may be obtained by taking a probabilistic approach (Cummins et al., 2014b). Specifically, given an $N$-dimensional feature space, $X \in \mathcal{R}^{N}$, if the underlying distribution of the features is denoted by $P(X)$, an estimate of the Probabilistic Acoustic Volume (PAV), $V_{\theta}$, can be obtained as the total volume of the space where $P(X)$ is greater than some threshold $\theta$ (refer Figure 10). i.e.,

$$
V_{\theta}=\int f(X) d X, \quad f(X)= \begin{cases}1, & P(X)>\theta \\ 0, & P(X) \leq \theta\end{cases}
$$




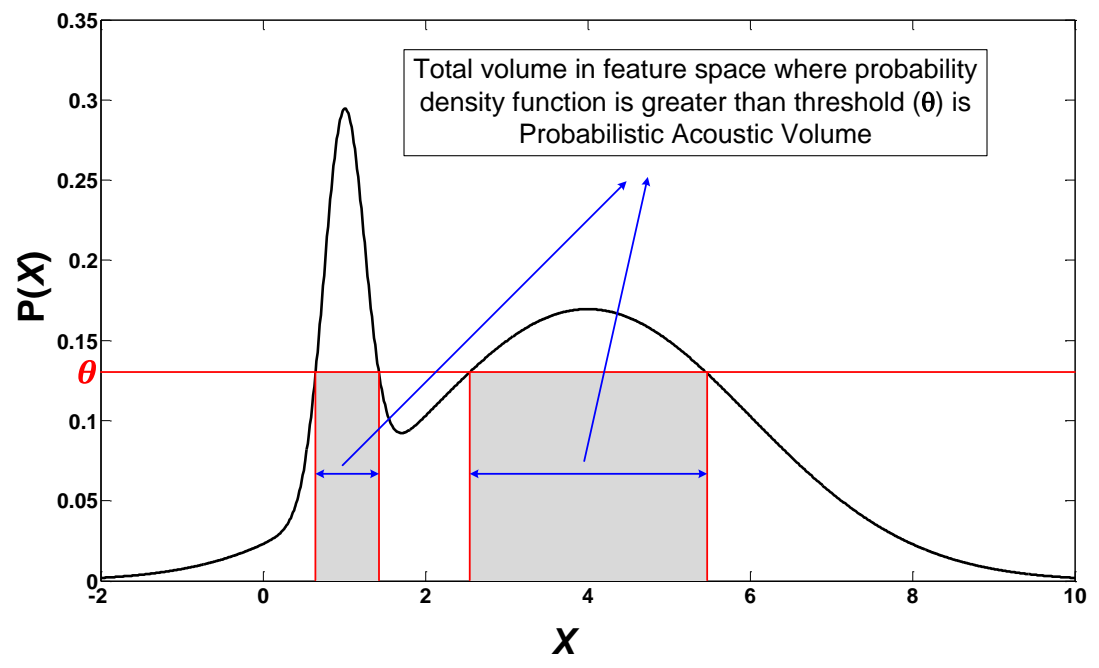

Figure 10: Sample 1-dimensional feature distribution with volume of feature space corresponding to probability higher than a threshold $-\theta$

A Monte Carlo approach can be used to estimate $V_{\theta}$. By rewriting (9) as (Cummins et al., 2014b):

$$
V_{\theta}=\int \hat{f}(X) P(X) d X
$$

where

$$
\hat{f}(X)=\left\{\begin{array}{cc}
\frac{1}{P(X)}, & P(X)>\theta \\
0, & \text { otherwise }
\end{array}\right.
$$

$V_{\theta}$ can be expressed as:

$$
V_{\theta}=E_{P}[\hat{f}(X)]
$$

where, $E_{P}[\cdot]$ denotes the expected value with respect to the probability density function $P(X)$. A Monte Carlo approximation of this value is given by

$$
\widehat{V}_{\theta}=\frac{1}{N} \sum_{x_{n} \sim P(X)} \hat{f}\left(x_{n}\right), \quad 1 \leq n \leq N
$$

where $x_{n}$ denotes the $n$-th random samples drawn from the feature distribution $P(X)$ and $N$ denotes the total number of such samples drawn (Andrieu et al., 2003). 
When a $\mathrm{GMM}, \mathcal{G}(X)$, is used to approximate $P(X), V_{\theta}$ can be estimated through a Monte Carlo approach (Cummins et al., 2014b). Specifically, given an $M$-mixture GMM, $N$ points are drawn from it by drawing $N_{i}$ points from each Gaussian component such that:

$$
N_{i} / N=\omega_{i}
$$

where $\omega_{i}$ is the weight associated with the $i^{\text {th }}$ component and

$$
\sum_{i} N_{i}=N
$$

The PAV estimate $\widehat{V}_{\theta}$, is the found by summing the fraction of points whose probability, evaluated using the $\mathrm{GMM}$ as $\mathcal{G}\left(x_{n}\right)$, is greater than the threshold $\theta$ (Figure 11);

$$
\widehat{V}_{\theta}=\frac{1}{N} \sum_{x_{n} \sim \mathcal{G}(X)} \varphi\left(x_{n}\right), \quad 1 \leq n \leq N
$$

where

$$
\varphi(X)=\left\{\begin{array}{cc}
\frac{1}{\mathcal{G}(X)}, & \mathcal{G}(X)>\theta \\
0, & \text { otherwise }
\end{array}\right.
$$

Note for the PAV results presented in this section, $\mathcal{G}(X)$ is the file-specific GMMs, Section 3.1, trained using MAP-adaptation, Section 2.3. 


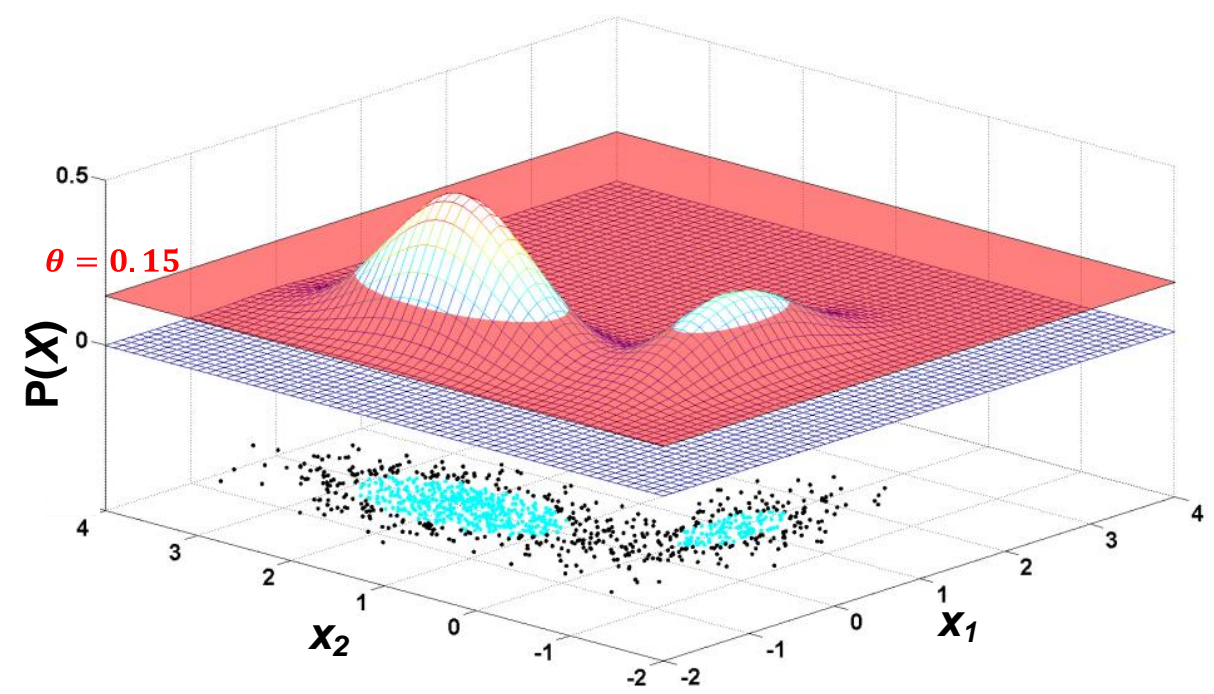

Figure 11: Example 2-mixture GMM of a synthetic $2 D$ feature space $\left(x_{1}, x_{2}\right.$, arbitrary 'feature values' for illustrative purposes) and points drawn from the mixtures of the GMM - black denotes points with probability less than $\theta=0.15$ and blue denotes points with probability greater than $\theta=0.15$.

PAV is more than just a simple measure of acoustic volume - it also offers an estimation of the concentration of the feature vectors in the feature space (Cummins et al., 2014b). As seen in Figure 10 and Figure 11 , selecting a value for $\theta$ is akin to picking a cross-section of the feature distribution and $V_{\theta}$ is an estimate of the cross-sectional volume in the feature space. By defining a series of thresholds, $\theta_{i}$ for $1 \leq i \leq L$, an array of probabilistic acoustic volumes, $\mathrm{V}$, herein referred to as the probabilistic acoustic volume profile (PAV profile), can be obtained which is characteristic of the concentration of the feature vectors in the feature space (refer Figure 12). Further, the PAV profile is expected to be more immune to the effects of outliers than GM-AV; given that outliers are by definition data points (feature vectors) of low probability, it can be expected that the PAV profile would not be distorted by outliers since a range of $\theta$ values are taken into consideration. 
(a)

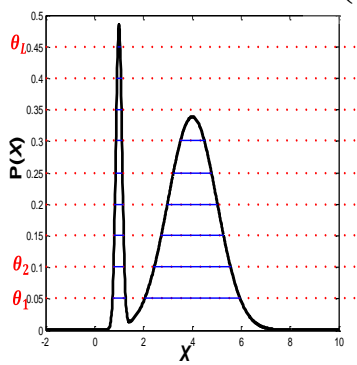

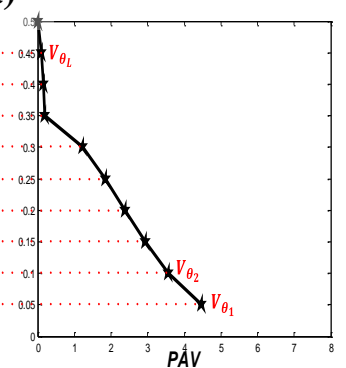

(b)

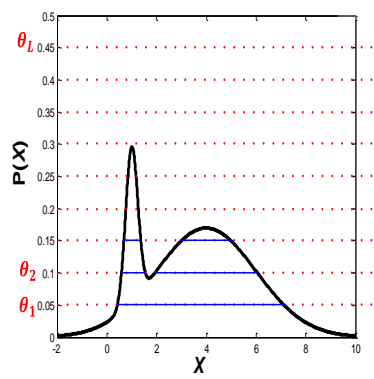

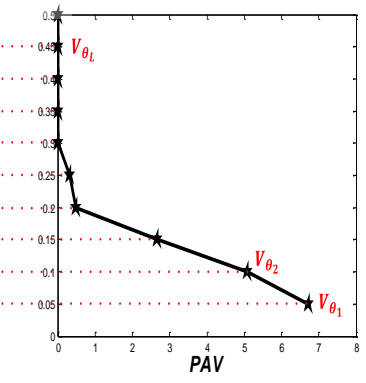

Figure 12: The shape of the PAV profile is representative of whether the feature vectors are tightly concentrated. (a) Estimate of probabilistic acoustic volume profile for an example 1-dimensional feature distribution reflecting two wide low density feature clusters exhibiting a low concentration of features.

(b) Estimate of probabilistic acoustic volume profile for a sample 1-dimensional feature distribution reflecting two small highly densely concentrated feature clusters

Insights into how the concentration of the feature vectors in the feature space change with increasing levels of speaker depression can be gained by viewing the correlation and effect size of the individual PAVprofile points (Figure 13). Figure 13(a) and Figure 13(b) show the correlation coefficients and effect size, respectively, for the AVEC-2013 PAV points for a 32 mix GMM. Note that in Figure 13(a) and Figure 13(b) white points indicate statistically insignificant points, light-grey indicates significance $(p<.05)$, dark-grey indicates moderate significance $(p<.01)$ and black indicates strong significance $(p<.001)$. Both plots have two distinct areas of statistical significance, $(-135 \leq$ $\log (\theta) \leq-110)$ and $(-100 \leq \log (\theta) \leq-85)$.

The negative correlations at the lower distributions $(-135 \leq \log (\theta) \leq-110)$ suggest that as depression increases there is comparatively less volume at these distributions. Conversely the positive correlation at the higher thresholds, $(-100 \leq \log (\theta) \leq-85)$ suggests with increasing levels of depression there is comparatively more volume at the higher thresholds. Figure $13 c$ shows an example of PAVprofiles for two speakers, one at a high level of depression and one at a low level of depression. It can be seen that the depressed speaker has a larger PAV at the higher thresholds and 
a smaller PAV at the lower thresholds. The crossover point of these two distributions matches the area of non-statistical significant PAV values in Figure 13a. and Figure 13b.

The statistical trends seen in Figure 13 suggest a fundamental change may occur in the distribution of the underlying feature vector concentration modelled in the speaker specific GMM's. Comparatively fewer PAV points at lower threshold and more PAV points at the higher thresholds indicate that the MFCC feature space becomes more tightly concentrated with increasing levels of depression. A similar set of results is seen for the Mundt-35 corpus, not plotted, although the statistical results, especially in the effect size, are not as strong. 


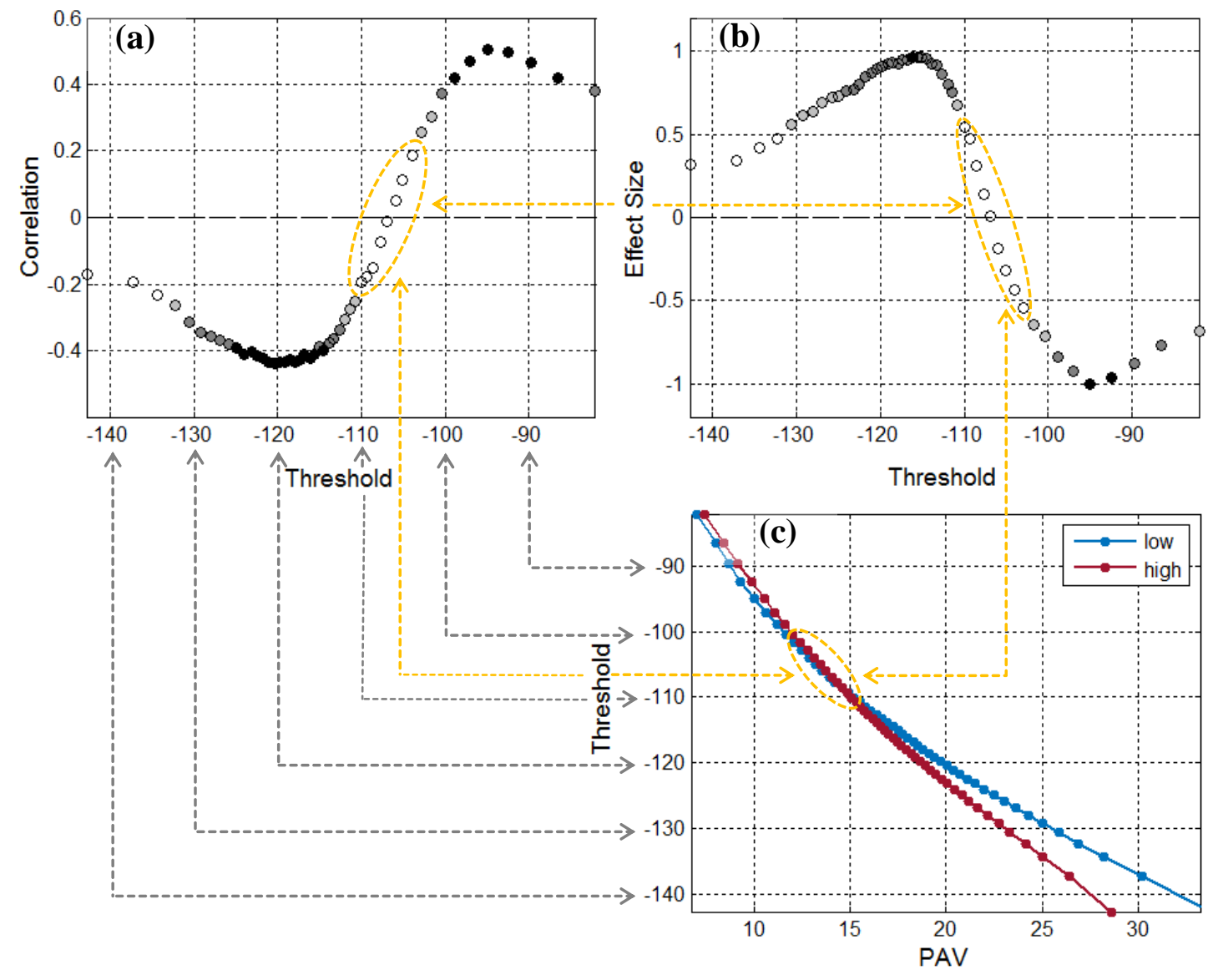

Figure 13: Effect of depression on each of the 50 PAV points extracted using a 32 GMM from AVEC-2013,

(a) correlations between the individual PAVprofile points and BDI; correlations found with $n=68$ files (b) effect size, found using $n=26$ files per class, of the individual PAVprofile points, note Light-Grey indicates significance (p<.05), Dark-Grey indicates moderate significance $(p<.01)$ and black indicates strong significance (p<.001), (c) An example of two PAVprofiles for speakers at a low level of depression (blue, $B D I<10)$ and a high level of depression (red, $B D I>18)$. Notice the yellow lines showing how the not significant correlation and effect size PAV points match up with the crossover area in the PAVprofiles.

Cummins et al. (2014b) show, for AVEC-2013, the steepness of a negative linear slope (PAVslope) was fitted to each of the profiles is statistically correlated with depression. This result provides further evidence with our previous assertion that the MFCC feature space becomes more tightly concentrated with increasing levels of depression. To test if this effect can be seen in the Mundt-35 corpus and noting the shape of the distribution of the PAVprofile in Figure 13c, an exponential curve 
is fitted to each PAVprofile and the resulting decay coefficient (PAVexp) tested for its suitability as a summary one-dimensional marker of depression.

Care needs to be taken when setting the PAV threshold points. If $\theta_{i}$ is set too low the majority of the distribution will be above the threshold and the resulting $V_{\theta_{i}}$ will not contain sufficient discriminatory information. Conversely if $\theta_{i}$ is too high the resulting $V_{\theta_{i}}$ will equal zero. It is possible to automatically ${ }^{5}$ set the threshold values using the log likelihood of the test utterances compared to a GMM. Given a set of speech frames $\boldsymbol{X}=\left\{\boldsymbol{x}_{1} \cdots \boldsymbol{x}_{T}\right\}$, and a GMM, $\mathcal{G}$, we first calculate the log likelihood of each frame in $X$ with respect to $\mathcal{G}$, i.e. $P(X \mid \mathcal{G})$, and we can estimate the distribution of $P(X)$ from a histogram representation of the resulting set of likelihoods. A PAVprofile, with $L=50$ was extracted per file using the percentiles of the estimated distribution; setting $\theta_{1}$ to the first percentile and $\theta_{L}$ to be the $99^{\text {th }}$ percentile (Figure 14). All threshold points in-between were set to every second percentile:

$$
\left\{\theta_{1}, \theta_{2}, \theta_{3}, \ldots, \theta_{49}, \theta_{50}\right\}=\left\{P(X)_{0.01}, P(X)_{0.03}, P(X)_{0.05}, \ldots, P(X)_{0.97}, P(X)_{0.99}\right\}
$$

The settings used in PAV extraction experiments reported in this section are; 100,000 points $(N)$ for the Monte Carlo approximation with a PAVprofile of 50 points were extracted per file. For AVEC2013, $P(X)$ for each GMM size was estimated from a the equivalent sized AVEC-2013 UBM (Section 3.2), using all voiced frames in the Homo Faber excerpt training and development partitions and a 1000 bin histogram. Whilst for Mundt-35 $P(X)$ for each GMM size was estimated, from the equivalent sized Mundt-35 UBM (Section 3.2), using all voiced frames in the Grandfather Passage and a 1000 bin histogram. To negate the effects of outliers in the profile, when calculating PAVexp, we removed all PAVprofiles points below the $25^{\text {th }}$ percentile for both AVEC-2013 and Mundt-35 and all PAVprofiles points above the $75^{\text {th }}$ percentile for Mundt-35, these values were determined experimentally.

\footnotetext{
${ }^{5}$ In (Cummins et al., 2014a) the $\theta$ values were set experimentally
} 


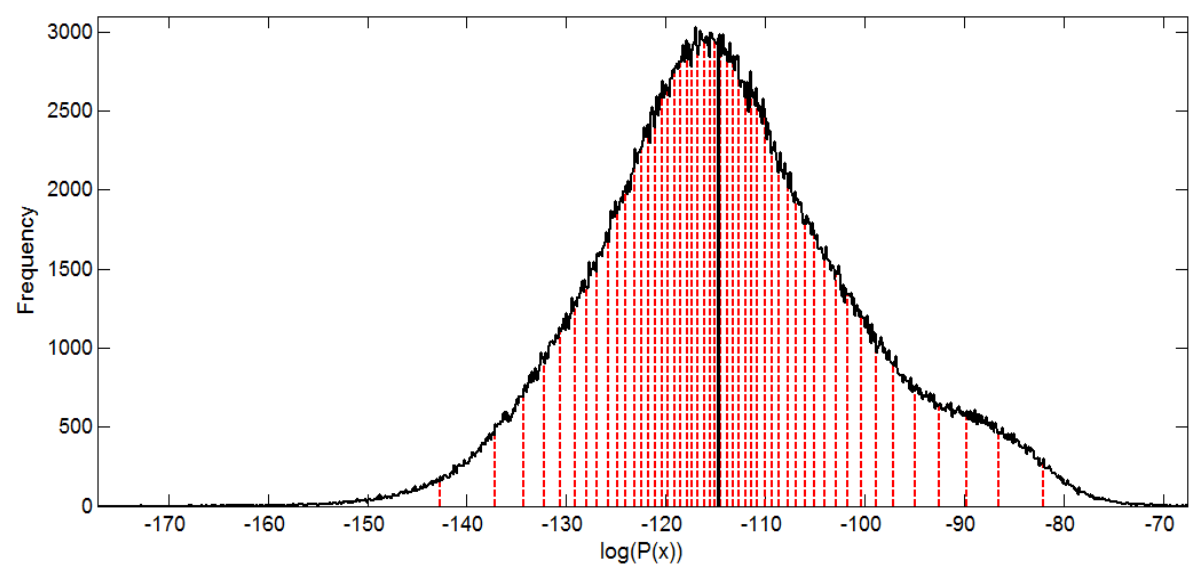

Figure 14: Distribution of $\boldsymbol{P}(\boldsymbol{X})$ estimated from the histogram formed from the log likelihood of all speech frames in the AVEC2013 training partitions with respect to a 32 mix UBM

As with PAVslope, (Cummins et al., 2014b), PAVexp is not simply a measure of acoustic volume, it is a measure of the spread of data in the feature space. Given the results presented in Cummins et al. (2014b) we expect a negative rate of decay increasing with increasing levels of speaker depression, indicating a more tightly concentrated feature space.

For AVEC-2013, the strong negative correlations, Table 8, show the negative exponential rate of decay decreasing with increasing levels of speaker depression, providing strong evidence that in this corpus there is indeed an increasing negative rate of decay with increasing levels of speaker depression. The effect size results $(|g|>1.0, p<.001)$ provide strong evidence for the suitability of this feature as a marker of depression for the AVEC-2013 corpus.

The Mundt-35 PAV results show similar trends to that corpus' AM results, with only the 8-mix GMM's providing both a significant correlation and effect size (Table 8). As the Mundt-35 $P(X)$ was estimated from a Mundt-35 UBM, it is possible these Mundt-35 results relate to the earlier discussion on UBM suitability (Section 4.3). If the $M \geq 32$ UBMs are potentially not a suitable portioning of the acoustic space for the Mundt-35 data, the reliability of the estimating thresholds points set using equation (18) would be compromised reducing the effectiveness of any subsequent results. 
Both the PAVexp results in combination with the PAVslope results presented in (Cummins et al., 2014b) provide strong evidence for our previous speculation that a speaker's MFCC feature space becomes more tightly concentrated with increasing levels of depression, with the resulting GMM distributions being taller and steeper. This speculation is also supported by the AWV results, Section 4.2. A concentrated feature, as indicated by comparatively fewer PAV points at lower thresholds, matches nicely with a decrease in localised variability.

Table 8: Statistical Analysis for PAVexp, calculated for both the AVEC-2013 and Mundt-35 Speaker Depression Corpora. Note only the $\boldsymbol{P}(\boldsymbol{X})$ percentiles 25:99 were used for AVEC2013 and 25:75 for Mundt-35. Correlations were found with $n=68$ files for AVEC-2013 and $n=118$ files for Mundt-35. Effect size results were found using $n=26$ files per class for AVEC-2013 and $n=43$ files per class for Mundt-35.

\begin{tabular}{|c|c|l||l|}
\hline Corpus & $\begin{array}{c}\text { GMM } \\
\text { Mix }\end{array}$ & $\begin{array}{c}\text { Correlation } \\
r_{s}\end{array}$ & $\begin{array}{c}\text { Effect Size } \\
g\end{array}$ \\
\hline & 8 & $-0.45^{* * *}$ & $1.22^{* * *}$ \\
& 16 & $-0.48^{* * *}$ & $1.29^{* * *}$ \\
AVEC-2013 & 32 & $-0.44^{* * *}$ & $1.23^{* * *}$ \\
& 64 & $-0.42^{* * *}$ & $1.18^{* * *}$ \\
& 128 & $-0.42^{* * *}$ & $1.18^{* * *}$ \\
& & & \\
& 8 & $-0.32^{* * *}$ & $0.45^{*}$ \\
Mundt-35 & 16 & $-0.26^{* *}$ & 0.26 \\
& 32 & $-0.27^{* *}$ & 0.31 \\
& 64 & $-0.26^{* *}$ & 0.24 \\
& 128 & $-0.23^{*}$ & 0.14 \\
\hline
\end{tabular}

When comparing PAV with GM-AV and ICdist, it can be seen that they do not estimate the same quantity (Figure 15). The GM-AV is an estimate of the hypervolume spanned by all mixture components of a GMM. ICdist is the arithmetic mean of the distances between mixtures and provides information on the closeness of the clusters in acoustic space. Whilst PAV on the other hand is independent of distances between feature clusters and is dependent only on the size and density of feature clusters and when combined into an array of PAV's offers insights into the underlying feature space concentration. However the combined GM-AV, ICdist and PAV results, which all show negative correlations with depression severity, Table 6, Table 7 and Table 8 as well as 
Figure 13, provide strong evidence for a decrease in acoustic variability - reduction in hypervolume, reduction in average distance between clusters and a more tightly concentrated feature space with increasing levels of speaker depression. Combining this result with the decreases in vowel space reported by Scherer et al. (2015), there is strong evidence that depression affects speech motor control, reducing the spread of phonetic events in acoustic space.

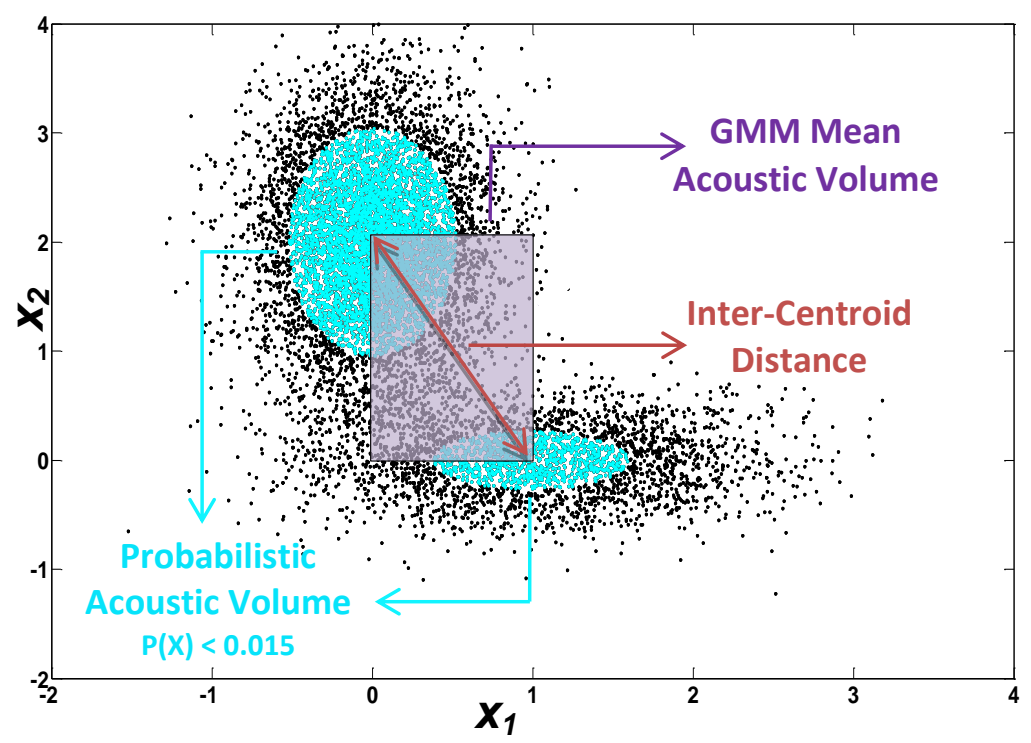

Figure 15: An example of a synthetic 2D feature space $\left(x_{1}, x_{2}\right.$, arbitrary 'feature values' for illustrative purposes) showing a comparison of Probabilistic Acoustic Volume (PAV), GMM mean acoustic volume (GM-AV) and Inter-Centroid Distance (ICdist) - Points drawn from example 2-mixture GMM, with blue denoting the points considered in computing PAV with probability greater than $\theta=0.15$. The violet box represents GM-AV computed from mixture means. The red double arrow represents the ICdist.

\section{Comparing Results across Corpora}

In Section 4, the AVEC-2013 results in general reveal stronger correlations and larger effect sizes between the acoustic space features and a speaker's level of depression when compared to Mundt35; this is most apparent in the Average Weighted Variance (AWV) and Acoustic Movement (AM) results - Table 3 and Table 5 respectively. This Section is a discussion on the differences in statistical significance between AVEC-2013 and Mundt-35 focusing on the AWV and AM features. 
It is possible that the differences seen between the two corpora could be due to; language differences, German (AVEC-2013) versus American English (Mundt-35); recording setup, headset connected to a laptop (AVEC-2013) versus telephone interface (Mundt-35); or differing speaker characteristics such as gender, age, height, and weight between the two datasets. Without suitable alternative data i.e. the same speakers present in AVEC-2013 recorded over a telephone interface, these speculations are hard to test. However, both channel and speaker characteristics have been shown to have strong confounding effects when performing automatic classification or prediction of speech affected by depression (Cummins et al., 2014a, 2013c, 2011; Sturim et al., 2011).

Another potential reason for the differences in statistical significance could be the shorter length of adaptation data used to generate the Mundt-35 speaker models (Section 3). However, re-running the AVEC-2013 AWV tests using only 50secs of adaptation data taken from the start of the Homo Faber passage resulted in only small changes from results found using the full passage (Table 9). Conversely, increasing the average amount of adaptation data per file, to 109.37 secs by adding extra speech tasks to the Grandfather Passage, does not significantly improve on the Mundt-35 AWV results (Table 9). We note that whilst the results in Table 9 are for either 32-mix (AVEC-2013) or 8-, 16-mix (Mundt-35) Gaussian Mixture Models (GMM), similar results were seen across the different sized GMM's. 
Table 9: Comparing statistical analysis of AWV's calculated using different amount of adaption data for both the AVEC-2013 and Mundt-35 Corpora. Correlations were found with $n=68$ files for AVEC-2013 and $n=118$ files for Mundt-35. Effect size results were found using $n=26$ files per class for AVEC-2013 and $n=43$ files per class for Mundt-35.

\begin{tabular}{|c|c|c|c|c|}
\hline Corpora & $\begin{array}{c}\text { Adaptation Data } \\
\text { (Average file length) }\end{array}$ & $\begin{array}{c}\text { GMM } \\
\text { Mix }\end{array}$ & $\begin{array}{c}\text { Correlation } \\
r_{s}\end{array}$ & $\begin{array}{c}\text { Effect Size } \\
g\end{array}$ \\
\hline AVEC-2013 & $\begin{array}{l}\text { "Homo Faber" } \\
\text { (204secs) }\end{array}$ & 32 & $-0.44 * * *$ & $0.69 *$ \\
\hline AVEC-2013 & $\begin{array}{l}\text { "Homo Faber" } \\
\text { (First 50secs) }\end{array}$ & 32 & $-0.43 * * *$ & $0.74^{* *}$ \\
\hline Mundt-35 & $\begin{array}{l}\text { Grandfather } \\
\text { (50secs) }\end{array}$ & $\begin{array}{l}8 \\
16\end{array}$ & $\begin{array}{l}-0.23^{*} \\
-0.19^{*}\end{array}$ & $\begin{array}{l}0.53^{*} \\
0.27\end{array}$ \\
\hline Mundt-35 & $\begin{array}{l}\text { Grandfather + Extra } \\
\text { (109.37secs) }\end{array}$ & $\begin{array}{l}8 \\
16\end{array}$ & $\begin{array}{l}-0.21^{*} \\
-0.15\end{array}$ & $\begin{array}{l}0.34 \\
0.30\end{array}$ \\
\hline
\end{tabular}

We speculate that the results in Table 9 show that, within limits, the amount of adaption data may not be as important as the paradigm the data was collected under. It has been suggested that the acoustic effects of depression may not be strongly reflected in "automatic" speech tasks such as counting, vocal exercises and short reading passages (Alpert et al., 2001; Calev et al., 1989; Ellgring and Scherer, 1996; Nilsonne et al., 1987; Stassen et al., 1991). This is due to a variety of different reasons such as the [lack of] cognitive effort required and practise effects, where improvements in speech quality could be due to familiarity with the passage as opposed to improvements in speech motor control. Changes in vocal behaviour, due to changes in speaker affect, are also consider to be topic dependent (Ellgring and Scherer, 1996; Scherer, 2003). This is particularly relevant to depression as depressed individuals show a specific failure to impair inhibitions relating to negative information (Goeleven et al., 2006). Work by Vanger et al. (1992) shows that increasing the negative content, in a semi-structured interview paradigm, can induce changes in vocal behaviour of depressed individuals regardless of their level of depression. 
Therefore, it is possible that the Grandfather Passage, designed to test speech fluency, doesn't evoke any emotional or motivational reader effects. The Homo Faber passage, whilst still being a read passage, is considerably longer than standard "automatic" speech readings such as the Grandfather or Rainbow passage. Due to this increase in passage length the acoustic effects of depression could be more strongly reflected in this passage due to the speaker's [lack of] motivation to complete and engage in the task as well as weakness and fatigue effects. Evidence of increased speaker fatigue can be seen in the analysis of the difference in the relative strength of the AM statistical analysis when using the last 50 s of the Homo Faber passage compared to the first 50s (Table 10). The Homo Faber passage was also chosen for inclusion in the AVEC-2013 corpus as being regarded as one of the most important and well known German novels, it would induce a natural interest and motivation to read and it would induce a stronger emotional response in the reader.

Table 10: Comparing statistical analysis of $A M^{\prime}$ 's calculated using different amount of adaption data for AVEC-2013. Correlations were found with $n=68$ files for AVEC-2013 and. Effect size results were found using $n=26$ files per class

\begin{tabular}{|c|l||c||c||c|}
\hline Corpora & $\begin{array}{l}\text { Adaptation Data } \\
\text { (Average file length) }\end{array}$ & $\begin{array}{c}\text { GMM } \\
\text { Mix }\end{array}$ & $\begin{array}{c}\text { Correlation } \\
r_{s}\end{array}$ & $\begin{array}{l}\text { Effect Size } \\
g\end{array}$ \\
\hline AVEC-2013 & $\begin{array}{l}\text { "Homo Faber" } \\
\text { First 50sec }\end{array}$ & 16 & -0.23 & 0.42 \\
AVEC-2013 & $\begin{array}{l}\text { "Homo Faber" } \\
\text { Last 50sec }\end{array}$ & 16 & $-0.29 *$ & $0.66^{*}$ \\
\hline$* p<.05$ & \multicolumn{2}{|l}{} \\
\hline
\end{tabular}

Adding weight to our speculation on file suitability is the results seen when re-running the Mundt-35 AM tests (Table 11). Using the participant answers to questions on either their current emotional and physical state as the evaluation utterance, we see stronger correlations and larger effect sizes when compared to those found for the Grandfather Passage - in the 8- and 16-mix results with the physical state showing higher correlations and a stronger effect size than the emotional state (Table 11). Note that the analyses performed in Table 10 and Table 11 were only performed on the AM variability measure. We speculate $\mathrm{AM}$ - as a measure of movement between acoustic regions as 
opposed to a measure relating to the size of acoustic regions - is more robust to variations caused by the different phonetic content in each evaluation file.

Table 11: Statistical test results for AM calculated for free response answer to "Feeling Physically" and "Feeling Emotionally" questions in Mundt 35 Speaker Depression Corpora. Note: no VAD was used in these calculations. Correlations were found with $n=118$ files and Effect size results were found using $n=43$ files per class.

\begin{tabular}{|c|c|c|c|c|}
\hline Corpora & $\begin{array}{c}\text { Adaptation Data } \\
\text { (Average file length) }\end{array}$ & $\begin{array}{l}\text { GMM } \\
\text { Mix }\end{array}$ & $\begin{array}{c}\text { Correlation } \\
r_{s} \\
\end{array}$ & $\begin{array}{c}\text { Effect Size } \\
g\end{array}$ \\
\hline Mundt-35 & $\begin{array}{l}\text { Grandfather } \\
\text { (50secs) }\end{array}$ & $\begin{array}{l}8 \\
16\end{array}$ & $\begin{array}{l}-0.23^{*} \\
-0.19^{*}\end{array}$ & $\begin{array}{l}0.53^{*} \\
0.27\end{array}$ \\
\hline Mundt-35 & $\begin{array}{l}\text { Feeling Emotionally } \\
\text { (46secs) }\end{array}$ & $\begin{array}{l}8 \\
16\end{array}$ & $\begin{array}{l}-0.32 * * * \\
-0.20^{*}\end{array}$ & $\begin{array}{l}0.67 * * \\
0.32\end{array}$ \\
\hline Mundt-35 & $\begin{array}{l}\text { Feeling Physically } \\
\text { (43secs) }\end{array}$ & $\begin{array}{l}8 \\
16\end{array}$ & $\begin{array}{l}-0.48 * * * \\
-0.39 * * *\end{array}$ & $\begin{array}{l}0.98^{* * *} \\
0.76^{* *}\end{array}$ \\
\hline
\end{tabular}

\section{Conclusion}

This is the first study which specifically analyses the acoustic space - the generalised distribution of phonetic events - of speech affected by depression. The use of Gaussian mixture models (GMM) in combination with Mel Frequency Cepstral Coefficients (MFCC) is widely used to classify either the presence or severity of depression or predict a speakers level of depression (Alghowinem et al., 2012; Cummins et al., 2013b, 2013c, 2011; Low et al., 2010; Sturim et al., 2011). Therefore we wanted to gain an understanding into how this commonly used acoustic model is affected by depression.

We hypothesised that, given reported reductions in both energy variance, and sub-band energy variability with increasing levels of speaker depression (Cummins et al., 2013a, 2013b; Kuny and Stassen, 1993; Quatieri and Malyska, 2012), the effects of depression manifest as a reduction in the spread of phonetic events in acoustic space. In testing our hypothesis we present a range of acoustic 
variability measures specific for the task including; Average Weighted Variance (AWV), Acoustic Movement (AM) and Acoustic Volume measures including the novel Probabilistic Acoustic Volume (PAV) method for robustly estimating Acoustic Volume.

In the MFCC feature space we observed a consistent trend of negative correlations in spectral variability across all sub-bands and $\Delta$ and $\Delta \Delta$ coefficients. This was a verification of a key finding reported on the Mundt-35 corpus in Cummins et al. (2013b), but an important one; observing these negative correlations suggests there will be a decrease in the extent of spectral variations captured when modelling this feature space using a GMM.

The $A W V$ results show there is a decrease in localised acoustic variability with increasing level of depression. This result supports our hypothesis providing strong evidence that similar phonetic events are more concentrated in Acoustic Space. This indicates there is less variety within similar acoustic sounds with increasing levels of speaker depression. The AWV findings verified and expanded on observations reported on the Mundt-35 corpus in Cummins et al. (2013b) and also support the PAV observations outlined below.

Results found using $A M$ show there is a decrease in acoustic trajectory with increasing levels of speaker depression. This result provides evidence there are less abrupt acoustic movements matching descriptions of speech affected by depression as sounding slurred. With a smoother acoustic trajectory there will be less distinction between different phonetic sounds. This indicates that that utterances affect by depression contain less spectral variability matching the MFCC feature space results.

Finally the Acoustic Volume measures provide strong evidence for a global reduction in acoustic volume. GMM Mean Acoustic Volume (GM-AV) results indicates the overall "size" of acoustic space reduces with increasing levels of speaker depression whilst the Inter-Centroid Distance (ICdist) measures indicate that similar phonetic events are closer to each other in Acoustic Space. The PAV 
shows the MFCC feature space becoming tightly concentrated with increasing levels of speaker depression and GMM distributions becoming taller and steeper, matching nicely with the AWV results. The GM-AV and PAV results expanded on key findings reported for the AVEC-2013 corpus in Cummins et al. (2014b).

The results presented in this paper indicate that depression affect speech motor control. The reductions in spectral variability match the intensity decay reported for dysarthric speech (Kent and Kim, 2003), and the reductions in acoustic variability measures match with the reductions in vowel space reported in both dysarthric speech (McRae et al., 2002; Orozco-Arroyave et al., 2014; Sapir et al., 2010) and speech affected by depression (Scherer et al., 2015). Future research will involve testing the acoustic variability measures on conditions which may affect speech motor control, including Parkinson's disease, post-traumatic stress disorder and suicidality.

All of our main findings were verified over two commonly used depression databases; the Audio/Visual Emotion Challenge and Workshop 2013 dataset and James Mundt 35-speaker database. Our results indicate that speech affected by depression can be characterised by a combination of three effects; (i) a reduction in variability in speaking effort (ii) a lack of animation and (iii) a reduction in the number of distinct speech sounds produced. Exploiting these changes is the next step in finding a speech based marker of depression with clinical utility.

Future research questions resulting from the work presented in this paper include quantifying the behaviour of mean shifts during maximum a posteriori (MAP) adaptation as the speaker's level of depression changes (Section 4.2). A key component in the acoustic modelling paradigm used in this paper is the Universal Background Model (UBM). The AM results (Section 4.3) indicate it may be beneficial to investigate methods for selecting suitable UBM training data to ensure adequately coverage of the broad acoustic landscape (Hasan and Hansen, 2011). Results in Section 5 indicate that successful identification of a speech-based marker of depression can be influenced in part by the paradigm the speech data was collected under. Further research is required to understand 
effects of patient engagement, at both emotional and motivational levels, when eliciting suitable speech segments for speech based analysis.

A key reason for analysing the effects of depression in the GMM-UBM paradigm is that it allows for supervector analysis and a subsequent range of transforms in this domain to help minimize unwanted variability. We have previously shown it is possible using vectors which represent solely the lack of acoustic variation - an array of PAV points - to match prediction performance with a range of well-known spectral based supervectors (Cummins et al., 2014b). Therefore future research will focus on finding suitable supervector representations and complementary transforms capable of taking advantage of the reductions in acoustic space variability seen in depressed speech.

In conclusion all results, verified over two commonly used depression corpora, give strong evidence for reductions in both local and global spread of phonetic events in acoustic space with increasing levels of speaker depression. These results advance the results presented in Trevino et al. (2011) that phonetic events are less distinct in speech affected by depression. Reductions in acoustic variability match nicely with reported reductions in vowels space (Scherer et al., 2015), as well as the flat, dull, slurred, breathy or tense voice qualities commonly associated with speech affect by depression. Results found when comparing the analysis between the two corpora indicate a need to gain further understanding into how best to elicit speech samples for depression detection. Finally, this study suggests a concrete direction for future research - designing classification or prediction systems which suitably use this variability information to enhance accuracy and robustness.

\section{Acknowledgements}

The work of Nicholas Cummins and Julien Epps is supported by National ICT Australia, funded by the Australian Government as represented by the Department of Broadband, Communication and the Digital Economy and the Australian Research Council through the ICT Centre of Excellence program. Julien Epps is also supported by the Australian Research Council through Discovery Projects 
DP110105240 and DP120100641. The work of Jarek Krajewski and Sebastian Schnieder is partly funded by the German Research Foundation (KR3698/4-1). The authors would like to thank Dr James Mundt for the use of his database. The collection of these data was made possible by a Small Business Innovation Research grant (R43MH068950: JC. Mundt, PI) supported by the United States National Institute of Mental Health.

\section{References}

Alghowinem, S., Goecke, R., Wagner, M., Epps, J., Breakspear, M., Parker, G., 2012. From Joyous to Clinically Depressed: Mood Detection Using Spontaneous Speech, in: Twenty-Fifth International FLAIRS Conference. Marco Island, Florida, pp. 141-146.

Alku, P., Backstrom, T., Vilkman, E., 2002. Normalized amplitude quotient for parametrization of the glottal flow. J. Acoust. Soc. Am. 112, 701-710.

Allianz Deutschland, RWI, 2011. Depression-Wie die Krankheit unsere Seele belastet. Published online at: https://www.allianz.com/staticresources/en/press/media/documents/v_1302621328000/allianz_report_depression.pdf.

Alpert, M., Pouget, E.R., Silva, R.R., 2001. Reflections of depression in acoustic measures of the patient's speech. J. Affect. Disord. 66, 59-69.

American-Psychiatric-Association, 2013. Diagnostic and statistical manual of mental disorders : DSMV., 5th ed. American Psychiatric Association, Washington, DC.

Andrieu, C., de Freitas, N., Doucet, A., Jordan, M., 2003. An Introduction to MCMC for Machine Learning. Mach. Learn. 50, 5-43. 
Balsters, M.J.H., Krahmer, E.J., Swerts, M.G.J., Vingerhoets, A.J.J.M., 2012. Verbal and Nonverbal Correlates for Depression: A Review. Curr. Psychiatry Rev. 8, 227-234.

Blais, M., Baer, L., 2010. Understanding Rating Scales and Assessment Instruments, in: Baer, L., Blais, M.A. (Eds.), Handbook of Clinical Rating Scales and Assessment in Psychiatry and Mental Health, Current Clinical Psychiatry. Humana Press, pp. 1-6.

Bocklet, T., Steidl, S., Elmar, N., Skodda, S., 2013. Automatic Evaluation of Parkinson 's Speech Acoustic , Prosodic and Voice Related Cues, in: Proceedings of Interspeech. Lyon, France, pp. 1149-1153.

Calev, A., Nigal, D., Chazan, S., 1989. Retrieval from semantic memory using meaningful and meaningless constructs by depressed, stable bipolar and manic patients. Br. J. Clin. Psychol. 28, $67-73$.

Caligiuri, M.P., Ellwanger, J., 2000. Motor and cognitive aspects of motor retardation in depression. J. Affect. Disord. 57, 83-93.

Campbell, W.M., 2008. A covariance kernel for SVM language recognition, in: Acoustics, Speech and Signal Processing, 2008. ICASSP 2008. IEEE International Conference on. IEEE, pp. 4141-4144.

Campbell, W.M., Sturim, D.E., Reynolds, D.A., Solomonoff, A., 2006. SVM Based Speaker Verification using a GMM Supervector Kernel and NAP Variability Compensation, in: Proceedings of ICASSP. pp. 97-100.

Christopher, G., MacDonald, J., 2005. The impact of clinical depression on working memory. Cogn. Neuropsychiatry 10, 379-399. 
Crawford, A.A., Lewis, S., Nutt, D., Peters, T.J., Cowen, P., O’Donovan, M.C., Wiles, N., Lewis, G., 2014. Adverse effects from antidepressant treatment: randomised controlled trial of 601 depressed individuals. Psychopharmacology (Berl). 231, 2921-2931.

Cummins, N., Epps, J., Ambikairajah, E., 2013a. Spectro-Temporal Analysis of Speech Affected by Depression and Psychomotor Retardation, in: Proceedings of ICASSP. IEEE, Vancouver, Canada, pp. 7542-7546.

Cummins, N., Epps, J., Breakspear, M., Goecke, R., 2011. An Investigation of Depressed Speech Detection: Features and Normalization, in: Proceedings of Interspeech. ISCA, Florence, Italy, pp. 2997-3000.

Cummins, N., Epps, J., Sethu, V., Breakspear, M., Goecke, R., 2013b. Modeling Spectral Variability for the Classification of Depressed Speech, in: Proceedings of Interspeech. ISCA, Lyon, France, pp. 857-861.

Cummins, N., Epps, J., Sethu, V., Krajewski, J., 2014a. Variability Compensation in Small Data: Oversampled Extraction of i-vectors for the Classification of Depressed Speech, in: Proceedings of ICASSP. IEEE, Florence, Italy, pp. 970-974.

Cummins, N., Joshi, J., Dhall, A., Sethu, V., Goecke, R., Epps, J., 2013c. Diagnosis of Depression by Behavioural Signals: A Multimodal Approach, in: Proceedings of the 3rd ACM International Workshop on Audio/Visual Emotion Challenge (AVEC'13). Barcelona, Spain, pp. 11-20.

Cummins, N., Scherer, S., Krajewski, J., Schnieder, S., Epps, J., Quatieri, T., 2015. A Review of Depression and Suicide Risk Assessment using Speech Analysis. Speech Commun. 71, 1-49. 
Cummins, N., Sethu, V., Epps, J., Krajewski, J., 2014b. Probabilistic Acoustic Volume Analysis for Speech Affected by Depression, in: Proceedings of Interspeech. ISCA, Singapore, pp. 12381242.

Cusin, C., Yang, H., Yeung, A., Fava, M., 2010. Rating Scales for Depression, in: Baer, L., Blais, M.A. (Eds.), Handbook of Clinical Rating Scales and Assessment in Psychiatry and Mental Health SE 2, Current Clinical Psychiatry. Humana Press, pp. 7-35.

Dehak, N., Kenny, P.J., Dehak, R., Dumouchel, P., Ouellet, P., 2011. Front-End Factor Analysis for Speaker Verification. IEEE Trans. Audio. Speech. Lang. Processing 19, 788-798.

Domenici, E., Willé, D.R., Tozzi, F., Prokopenko, I., Miller, S., McKeown, A., Brittain, C., Rujescu, D., Giegling, I., Turck, C.W., Holsboer, F., Bullmore, E.T., Middleton, L., Merlo-Pich, E., Alexander, R.C., Muglia, P., 2010. Plasma Protein Biomarkers for Depression and Schizophrenia by Multi Analyte Profiling of Case-Control Collections. PLoS One 5 (2), e9166.

Ellgring, H., Scherer, K., 1996. Vocal indicators of mood change in depression. J. Nonverbal Behav. 20, 83-110.

Eyben, F., Wollmer, M., Schuller, B., 2010. Opensmile: the munich versatile and fast open-source audio feature extractor, in: Proceedings of the International Conference on Multimedia. ACM, Firenze, Italy, pp. 1459-1462.

Faries, D., Herrera, J., Rayamajhi, J., DeBrota, D., Demitrack, M., Potter, W.Z., 2000. The responsiveness of the Hamilton Depression Rating Scale. J. Psychiatr. Res. 34, 3-10.

Flint, A.J., Black, S.E., Campbell-Taylor, I., Gailey, G.F., Levinton, C., 1993. Abnormal speech articulation, psychomotor retardation, and subcortical dysfunction in major depression. J. Psychiatr. Res. 27, 309-319. 
France, D.J., Shiavi, R.G., Silverman, S., Silverman, M., Wilkes, M., 2000. Acoustical properties of speech as indicators of depression and suicidal risk. IEEE Trans. Bio-Eng, 47, 829-837.

Girard, J.M., Cohn, J.F., Mahoor, M.H., Mavadati, S.M., Hammal, Z., Rosenwald, D.P., 2013. Nonverbal social withdrawal in depression: Evidence from manual and automatic analyses. Image Vis. Comput. 32, 641-647.

Godino-Llorente, J.I., Gómez-Vilda, P., Blanco-Velasco, M., 2006. Dimensionality reduction of a pathological voice quality assessment system based on Gaussian mixture models and shortterm cepstral parameters. IEEE Trans. Biomed. Eng. 53, 1943-53.

Goeleven, E., De Raedt, R., Baert, S., Koster, E.H.W., 2006. Deficient inhibition of emotional information in depression. J. Affect. Disord. 93, 149-157.

Hall, J.A., Harrigan, J.A., Rosenthal, R., 1995. Nonverbal behavior in clinician-patient interaction. Appl. Prev. Psychol. 4, 21-37.

Hasan, T., Hansen, J.H.L., 2011. A study on universal background model training in speaker verification. Audio, Speech, Lang. Process. IEEE Trans. 19, 1890-1899.

Helfer, B.S., Quatieri, T.F., Williamson, J.R., Mehta, D.D., Horwitz, R., Yu, B., 2013. Classification of depression state based on articulatory precision, in: Proceedings of Interspeech. ISCA, Lyon, France, pp. 2172-2176.

Hönig, F., Batliner, A., Nöth, E., Schnieder, S., Krajewski, J., 2014. Automatic Modelling of Depressed Speech: Relevant Features and Relevance of Gender, in: Proceedings of Interspeech. Singapore, pp. $1248-1252$. 
Horwitz, R., Quatieri, T.F., Helfer, B.S., Yu, B., Williamson, J.R., Mundt, J., 2013. On the relative importance of vocal source, system, and prosody in human depression, in: Body Sensor Networks (BSN), 2013 IEEE International Conference on. Cambridge, MA, USA, pp. 1-6.

Joormann, J., Gotlib, I.H., 2008. Updating the Contents of Working Memory in Depression: Interference From Irrelevant Negative Material. J. Abnorm. Psychol. 117, 182-192.

Joshi, J., Goecke, R., Alghowinem, S., Dhall, A., Wagner, M., Epps, J., Parker, G., Breakspear, M., 2013. Multimodal Assistive Technologies for Depression Diagnosis and Monitoring. J. Multimodal User Interfaces 7, 217-228.

Kaya, H., Eyben, F., Salah, A.A., 2014. CCA based Feature Selection with Application to Continuous Depression Recognition from Acoustic Speech Features, in: 2014 IEEE Int. Conf. on Acoustics, Speech and Signal Processing (ICASSP). Florence, Italy, pp. 3757-3761.

Kenny, P., Ouellet, P., Dehak, N., Gupta, V., Dumouchel, P., 2008. A Study of Inter-Speaker Variability in Speaker Verification. Audio, Speech, Lang. Process. IEEE Trans. 16, 980 - 988.

Kent, R.D., 2000. Research on speech motor control and its disorders: A review and prospective. J. Commun. Disord. 33, 391-428.

Kent, R.D., Kim, Y.-J., 2003. Toward an acoustic typology of motor speech disorders. Clin. Linguist. Phon. 17, 427-445.

Kessler, R.C., Berglund, P., Demler, O., Al, E., 2003. The Epidemiology of Major Depressive Disorder: Results from the National Comorbidity Survey Replication (NCS-R). JAMA 289 (23), 3095-3105.

Kikuchi, T., Suzuki, T., Uchida, H., Watanabe, K., Mimura, M., 2012. Coping strategies for antidepressant side effects: an Internet survey. J. Affect. Disord. 143, 89-94. 
Krishnamurthy, N., Hansen, J., 2009. Babble noise: modeling, analysis, and applications. Audio, Speech, Lang. Process. IEEE Trans. 17, 1394-1407.

Kua, J.M.K., Epps, J., Nosratighods, M., Ambikairajah, E., Choi, E., 2011. Using clustering comparison measures for speaker recognition, in: Acoustics, Speech and Signal Processing (ICASSP), 2011 IEEE International Conference on. pp. 5452-5455.

Kuny, S., Stassen, H.H., 1993. Speaking behavior and voice sound characteristics in depressive patients during recovery. J. Psychiatr. Res. 27, 289-307.

Low, L.S.A., Maddage, M.C., Lech, M., Sheeber, L.B., Allen, N.B., 2011. Detection of Clinical Depression in Adolescents; Speech During Family Interactions. Biomed. Eng. IEEE Trans. 58, $574-586$.

Low, L.S.A., Maddage, N.C., Lech, M., Sheeber, L., Allen, N., 2010. Influence of acoustic low-level descriptors in the detection of clinical depression in adolescents, in: Proceedings of ICASSP. IEEE, Dallas, Texas, pp. 5154-5157.

Maust, D., Cristancho, M., Gray, L., Rushing, S., Tjoa, C., Thase, M.E., 2012. Chapter 13 - Psychiatric rating scales, in: Michael J. Aminoff, F.B., Dick, F.S. (Eds.), Handbook of Clinical Neurology. Elsevier, pp. 227-237.

McRae, P.A., Tjaden, K., Schoonings, B., 2002. Acoustic and perceptual consequences of articulatory rate change in Parkinson disease. J. Speech. Lang. Hear. Res. 45, 35-50.

Mitchell, A.J., Vaze, A., Rao, S., 2009. Clinical diagnosis of depression in primary care: a metaanalysis. Lancet 374, 609-619. 
Mundt, J.C., Snyder, P.J., Cannizzaro, M.S., Chappie, K., Geralts, D.S., 2007. Voice acoustic measures of depression severity and treatment response collected via interactive voice response (IVR) technology. J. Neurolinguistics 20, 50-64.

Mundt, J.C., Vogel, A.P., Feltner, D.E., Lenderking, W.R., 2012. Vocal Acoustic Biomarkers of Depression Severity and Treatment Response. Biol. Psychiatry 72, 580 -587.

Myers, J.L., Well, A., Lorch, R.F., 2010. Research design and statistical analysis, Third. ed. Routledge, New York, NY, USA.

Nilsonne, A., Sundberg, J., Ternstrom, S., Askenfelt, A., 1987. Measuring the rate of change of voice fundamental frequency in fluent speech during mental depression. J. Acoust. Soc. Am. 83, 716728.

Olesen, J., Gustavsson, A., Svensson, M., Wittchen, H.-U., Jönsson, B., Group, on behalf of the C. study, Council, the E.B., 2012. The economic cost of brain disorders in Europe. Eur. J. Neurol. $19,155-162$.

Orozco-Arroyave, J.R., Belalcázar-Bolaños, E.A., Arias-Londoño, J.D., Vargas-Bonilla, J.F., Haderlein, T., Nöth, E., 2014. Phonation and Articulation Analysis of Spanish Vowels for Automatic Detection of Parkinson's Disease, in: Sojka, P., Horák, A., Kopeček, I., Pala, K. (Eds.), Text, Speech and Dialogue, Lecture Notes in Computer Science. Springer International Publishing, pp. $374-381$.

$\varnothing$ stergaard, S.D., Jensen, S.O.W., Bech, P., 2011. The heterogeneity of the depressive syndrome: when numbers get serious. Acta Psychiatr. Scand. 124, 495-496.

Quatieri, T.F., Malyska, N., 2012. Vocal-Source Biomarkers for Depression: A Link to Psychomotor Activity, in: Proceedings of Interspeech. ICSA, Portland, USA, pp. 1059-1062. 
Reynolds, D.A., Quatieri, T.F., Dunn, R.B., 2000. Speaker Verification Using Adapted Gaussian Mixture Models. Digit. Signal Process. 10, 19-41.

Reynolds, D.A., Rose, R.C., 1995. Robust text-independent speaker identification using Gaussian mixture speaker models. Speech Audio Process. IEEE Trans. 3, 72-83.

Rosenthal, R., 1994. Parametric Measures of Effect Size., in: Cooper, H., Hedges, L.V. (Eds.), The Handbook of Research Synthesis. Russell Sage Foundation, New York, NY, US, pp. 231-244.

Sapir, S., Ramig, L.O., Spielman, J.L., Fox, C., 2010. Formant centralization ratio: a proposal for a new acoustic measure of dysarthric speech. J. Speech. Lang. Hear. Res. 53, 114-25.

Scherer, K.R., 2003. Vocal communication of emotion: A review of research paradigms. Speech Commun. 40, 227-256.

Scherer, S., Morency, L.P., Gratch, J., Pestian, J., 2015. Reduced Vowel Space is a Robust Indicator of Phychological Distress: A Cross-Corpus Analysis, in: Proceedings of ICASSP. pp. 4789-4793.

Scherer, S., Stratou, G., Gratch, J., Morency, L., 2013. Investigating Voice Quality as a SpeakerIndependent Indicator of Depression and PTSD, in: Proceedings of Interspeech. ISCA, Lyon, France, pp. 847-851.

Scherer, S., Stratou, G., Lucus, G., Mahmoud, M., Boberg, J., Gratch, J., Rizzo, A. (Skip), Morency, L.P.L.P., Lucas, G., 2014. Automatic Audiovisual Behavior Descriptors for Psychological Disorder Analysis. Image Vis. Comput. 32, 1-21.

Schmidt, H.D., Shelton, R.C., Duman, R.S., 2011. Functional Biomarkers of Depression: Diagnosis, Treatment, and Pathophysiology. Neuropsychopharmacology 36, 2375-2394. 
Schuller, B., Batliner, A., Steidl, S., Seppi, D., 2011. Recognising realistic emotions and affect in speech: State of the art and lessons learnt from the first challenge. Speech Commun. 53, 10621087.

Schuller, B., Steidl, S., Batliner, A., Burkhardt, F., Devillers, L., Müller, C., Narayanan, S., 2013. Paralinguistics in speech and language; State-of-the-art and the challenge. Comput. Speech Lang. 27, 4-39.

Sethu, V., Epps, J., Ambikairajah, E., 2014. Speech based emotion recognition, in: Ogunfunmi, T., Togneri, R., Narasimhai, M. (Eds.), Speech and Audio Processing for Coding Enhancement and Recognition. Springer, New York, pp. 197-228.

Skodda, S., Grönheit, W., Schlegel, U., 2012. Impairment of vowel articulation as a possible marker of disease progression in Parkinson's disease. PLoS One 7, e32132.

Skodda, S., Visser, W., Schlegel, U., 2011. Vowel Articulation in Parkinson's Disease. J. Voice 25, 467472.

Sobin, C., Sackeim, H.A., 1997. Psychomotor symptoms of depression. Am J Psychiatry 154, 4-17.

Stassen, H.H., Albers, M., Püschel, J., Scharfetter, C., Tewesmeier, M., Woggon, B., 1995. Speaking behavior and voice sound characteristics associated with negative schizophrenia. J. Psychiatr. Res. 29, 277-296.

Stassen, H.H., Bomben, G., Gunther, E., 1991. Speech characteristics in depression. Psychopathology $24,88-105$.

Stassen, H.H., Kuny, S., Hell, D., 1998. The speech analysis approach to determining onset of improvement under antidepressants. Eur. Neuropsychopharmacol. 8, 303-310. 
Steiger, A., Kimura, M., 2010. Wake and sleep EEG provide biomarkers in depression. J. Psychiatr. Res. 44, 242-252.

Sturim, D., Torres-Carrasquillo, P.A., Quatieri, T.F., Malyska, N., McCree, A., 2011. Automatic Detection of Depression in Speech Using Gaussian Mixture Modeling with Factor Analysis, in: Proceedings of Interspeech. ISCA, Florence, Italy, pp. 2983-2986.

Sullivan, G.M., Feinn, R., 2012. Using Effect Size-or Why the P Value Is Not Enough. J. Grad. Med. Educ. 4, 279-282.

Trevino, A., Quatieri, T., Malyska, N., 2011. Phonologically-based biomarkers for major depressive disorder. EURASIP J. Adv. Signal Process. 2011, 1-18.

Ustun, T.B., 2004. Global burden of depressive disorders in the year 2000. Br. J. Psychiatry 184, 386392.

Valstar, M., Schuller, B., Smith, K., Eyben, F., Jiang, B., Bilakhia, S., Schnieder, S., Cowie, R., Pantic, M., 2013. AVEC 2013: The Continuous Audio/Visual Emotion and Depression Recognition Challenge, in: Proceedings of the 3rd ACM International Workshop on Audio/Visual Emotion Challenge (AVEC'13). Barcelona, Spain, pp. 3-10.

Vanger, P., Summerfield, A.B., Rosen, B.K., Watson, J.P., 1992. Effects of communication content on speech behavior of depressives. Compr. Psychiatry 33, 39-41.

Wertz, R.T., LaPointe, L.L., Rosenbek, J.C., 1984. Apraxia of speech in adults: The disorder and its management. Grune \& Stratton, Orlando.

Williamson, J., Quatieri, T., Helfer, B., Ciccarelli, G., Mehta, D.D., 2014. Vocal and Facial Biomarkers of Depression based on Motor Incoordination and Timing, in: Proceedings of the 4th ACM 
International Workshop on Audio/Visual Emotion Challenge (AVEC'14). ACM, Orlando, Florida, USA, pp. 65-72.

Williamson, J.R., Quatieri, T.F., Helfer, B.S., Horwitz, R., Yu, B., Mehta, D.D., 2013. Vocal Biomarkers of Depression Based on Motor Incoordination, in: Proceedings of the 3rd ACM International Workshop on Audio/Visual Emotion Challenge. Barcelona, Spain, pp. 41-48.

World Health Organisation, 2004. Prevention of mental disorders. Geneva. Published online at: http://www.who.int/mental_health/publications/prevention_mh_2004/en/ 